\title{
Finite-order hydrodynamic model determination for wave energy applications using moment-matching
}

\author{
Nicolás Faedo*, Yerai Peña-Sanchez, John V. Ringwood \\ Centre for Ocean Energy Research, Maynooth University, Maynooth, Co. Kildare, Ireland
}

\section{A R T I C L E I N F O}

\section{Keywords:}

Radiation forces

Parametric form

Model order reduction

Moment-matching

Frequency-domain identification

\begin{abstract}
A B S T R A C T
The motion of a Wave Energy Converter (WEC) can be described in terms of an integro-differential equation, which involves a convolution product. The convolution term, which accounts for the radiation forces, represents a computational and representational drawback both for simulation, and analysis/design of control strategies. Several studies attempt to find a suitable finite parametric form that approximates the radiation impulse response, to express the equation of motion in the time-domain by a state-space representation. Ideally, this approximated parametric model should behave as closely as possible to the system under analysis, particularly at key frequencies, such as the resonant frequency of the device. This study presents a method to obtain a parametric model of both the force-to-motion dynamics and/or the radiation force convolution term, based on moment-matching. Recent advances in moment-matching, allow the computation of a model that exactly matches the frequency response of the original system at the chosen frequencies, while enforcing specific physical properties of the device, depicting a robust and efficient method to compute a state-space representation for the dynamics of a WEC. The potential of the algorithm is illustrated by numerical examples, and the approximation error is shown to be monotonically decreasing with increasing model order.
\end{abstract}

\section{Introduction}

Boundary Element Methods (BEM) are commonly used to calculate the hydrodynamic parameters of wave energy converters and, more generally, of various marine structures. While limited by the linear nature of potential flow theory, the speed with which numerical simulation may be performed when compared to other simulation methods, such as computational fluid dynamics or smoothed particle hydrodynamics, makes BEM a common choice to compute hydrodynamic parameters for a given WEC (Penalba et al., 2017a). Within the wave energy community, the most-widely used BEMs include the commercially available WAMIT (Newman and Lee, 2002) and the opensource NEMOH (Babarit and Delhommeau, 2015) numerical codes. However, one of the major drawbacks of BEMs is that the results are computed in frequency-domain and, hence, can only charaterise the steady-state motion of the WEC under analysis.

A more comprehensive dynamic modelling approach can be considered, using a time-domain representation of the motion of a WEC, in terms of the well-known Cummins' equation (Cummins, 1962). Moreover, a direct relationship between Cummins' equation and the hydrodynamic frequency-domain data (typically produced by WAMIT/ NEMOH), is given in (Ogilvie, 1964) (see Section 3 for further details).
The resulting time-domain dynamical model is an integro-differential equation, which contains a convolution term accounting for the fluid memory effects associated with radiation forces acting on a body.

Such a convolution operation usually represents a drawback, for two major reasons. Firstly, the direct computation of the convolution in a time-domain simulation scheme is computationally demanding. Secondly, such a term is inconvenient for the analysis and design of control systems, since modern (linear) control strategies are usually based on the availability of a state-space representation. Indeed, the vast majority of the optimal control techniques considered in the literature, which attempt to maximise the energy absorption of WECs, require a state-space approximation of the convolution term (Faedo et al.), with some notable exceptions, such as (Bacelli and Ringwood, 2015) and (Faedo et al.). This leads to the requirement for a suitable parametric approximation to the convolution term.

Several methods have been proposed in the literature to approximate the radiation convolution term, in terms of a linear time-invariant state-space representation. Noteworthy studies that provide a review on these multiple approximation methods, include (Taghipour et al., 2008), (Unneland, 2007) and (Roessling and Ringwood, 2015). These methodologies can be divided into two broad categories: time-domain and frequency-domain methods. A brief discussion on both approaches

\footnotetext{
* Corresponding author

E-mail address: nicolas.faedo.2017@mumail.ie (N. Faedo).
} 
is given in the following.

Time-domain methods use impulse response data, which is usually generated (via the inverse Fourier transform) from the frequency-domain data computed by BEMs, mainly due to the computational effort required to compute the time-domain response directly. Studies that consider a time-domain formulation to obtain a state-space representation of the radiation convolution term include, for example, (Yu and Falnes, 1995), (Hatecke, 2015) and (Kristiansen et al., 2005). It is important to note that, in some studies (such as (Kristiansen et al., 2005)), an initial higher-order approximation is determined, followed by a model order reduction stage. In the particular case of (Kristiansen et al., 2005), model order reduction via balanced truncation (Antoulas, 2005 ) is considered for the second stage. An extensive discussion on this two-phase approximation procedure can be found in (Unneland, 2007).

Frequency-domain parameterisation methods attempt to compute a parametric model directly from the frequency-domain data calculated by BEMs. As discussed in (Taghipour et al., 2008), these methods can be divided into several categories. Some studies, such as (Sutulo and Soares, 2005) and (Xia et al., 1998), compute a parametric form for each hydrodynamic parameter (i.e. added mass and radiation damping) separately, and then reconstruct the corresponding radiation impulse response function. An alternative, and the most-widely used, formulation finds a state-space form for the radiation dynamics directly, based on its frequency response, which can be readily computed using the hydrodynamic characteristics of the device (see, for example, (Pérez and Fossen, 2008), (Jordán and Beltrán-Aguedo, 2004), (Holappa and Falzarano, 1998), (McCabe et al., 2005) and (Ø. et al., 2014)). A further alternative approach considered, for example, in (Perez and Lande, 2006), is to compute a state-space representation of the complete forceto-motion dynamics, instead of finding only a parameterisation of the radiation convolution term. In this case, the physical notion of each component of the state vector is somewhat lost, though the outputs still represent physical variables. Note that, with this overall formulation, the order of the state-space representation obtained is usually lower (for equal fidelity of the overall model) than first computing a parametric form for the convolution term separately, and then embedding it into Cummins' equation. In fact, this last approach always requires two additional elements in the state-space representation to describe the force-to-motion dynamics (i.e. position and velocity of the device). This difference between both methodologies can be of particular importance, for example, in model-based optimal control design for WECs, where an excessive number of model states can render an energymaximising optimal controller unsuitable for real-time applications (the reader is referred to (Faedo et al.) for further details).

Regardless of the strategy chosen, a suitable parametric form, for wave energy applications, should represent either the force-to-motion dynamics or the radiation force convolution term (to incorporate into Cummins' equation), such that the behaviour of the approximated model is as close as possible to the target dynamics in a given (input) frequency range of interest. Furthermore, there are key frequencies, such as the resonant frequency of the device under analysis, that have a strong impact on the system dynamics. Ideally, the response of the approximated model should "match" the device dynamics at these specific key frequencies while, at the same time, approximating the behaviour of the target device over a frequency range of interest. Such a range is usually selected accordingly to the spectrum of the excitation force, as discussed in Section 4.1. Another important feature of a suitable identification technique is that the approximation error should decrease monotonically with increasing model order. This ensures that a higher number of elements to represent the state of the approximated model always decreases the approximation error. This is not always the case, as already reported in (Bertram et al., 2001), (Pérez and Fossen, 2008) and (Perez and Fossen, 2009) and can make the choice of approximating order somewhat haphazard. In particular (Pérez and Fossen, 2008; Perez and Fossen, 2009), report that the frequency- domain approximation algorithm studied suffers from stability issues when considering high-order approximations, although they declare that the approximation error will decrease significantly before expecting any increase in such an error value.

In light of the ideal characteristics described above, this paper proposes an approximation technique based on recent advances on model order reduction by moment-matching, developed over several studies, such as (Astolfi, 2010; Scarciotti and Astolfi, 2016a, 2017a, 2017b). As thoroughly discussed in Section 2, moment-matching methods are based on the idea of interpolating a certain number of points on the complex plane called moments. Moments have a direct relationship with the frequency-response of the dynamical system. In fact, a model reduced via moment-matching is such that its transfer function matches the behaviour of the transfer function of the target system at specific interpolation points (i.e. the moments). This is indeed one of the ideal features required in wave energy applications: a model, reduced by moment-matching, can be designed to match exactly the frequency response of the device under analysis, at specific key frequencies. Such an approach has several advantages compared to an identification plus reduction technique (as considered, for example, in (Kristiansen et al., 2005)): there is no need to perform a higher order identification of the system, since the reduced order model matches the moments of the unknown system, it is not just the result of a low-order identification but it actually retain some key properties of the system under analysis (Scarciotti and Astolfi, 2017b). Furthermore, given this intuitive property of the moment-matching approach, essential physical properties of the device can be enforced on the reduced order model, such as input-output stability. Note that stability is not usually guaranteed by current radiation force impulse response identification algorithms, so that several "fixes" have been proposed (further discussed in Section 5).

We note that the process of determining a finite-order dynamical model from 'frequency response' data points can alternatively be termed system identification (determining a model from frequency response data), or model-order reduction, where the starting model order is effectively the number of frequency points available. In the paper, we use the term 'model order reduction', in order to be more consistent with previous literature on moment-matching.

The remainder of the paper is organised as follows. In Section 2, the definition of moment, and the theoretical framework behind model order reduction by moment-matching, is introduced. Section 3 recalls the equation of motion of a floating body, in both frequency and time domain formulations. In Section 4, moment-based model order reduction is applied to the WEC case, to obtain a suitable parametric form for both the complete force-to-motion dynamics, and just the convolution term of Cummins' equation. Section 5 presents numerical examples of the proposed technique, using frequency-domain data for particular WEC devices. Finally, a discussion and concluding remarks are presented in Section 6.

\subsection{Notation and preliminaries}

Standard notation is considered through this study, with any exceptions detailed in this section. $\mathbb{R}^{+}\left(\mathbb{R}^{-}\right)$denotes the set of non-negative (non-positive) real numbers. $\mathbb{C}^{0}$ denotes the set of pure-imaginary complex numbers and $\mathbb{C}^{-}$denotes the set of complex numbers with a negative real part. The symbol 0 stands for any zero element, dimensioned according to the context. The symbol $\mathbb{U}_{n}$ denotes an order $n$ identity matrix. The spectrum of a matrix $A \in \mathbb{R}^{n \times n}$, i.e. the set of its eigenvalues, is denoted as $\sigma(A)$. The symbol $\oplus$ denotes the direct sum of $n$ matrices, i.e. $\oplus_{i=1}^{n} A_{i}=\operatorname{diag}\left(A_{1}, A_{2}, \ldots, A_{n}\right)$. The notation $\mathfrak{R}\{z\}$ and $\Im\{z\}$, with $z \in \mathbb{C}$, stands for the real-part and the imaginary-part operators, respectively. The expression $\|x\|_{2}$, with $x \in \mathbb{C}^{n \times 1}$, denotes the $\ell^{2}$-norm of the complex-valued vector $x$. The Kronecker product between two matrices $M_{1} \in \mathbb{R}^{n \times m}$ and $M_{2} \in \mathbb{R}^{p \times q}$ is denoted as $M_{1} \otimes M_{2}$ $\in \mathbb{R}^{n p \times m q}$, while the convolution between two functions $f(t)$ and $g(t)$ 
over a finite range $[0, t]$, i.e. $\int_{0}^{t} f(\tau) g(t-\tau) d \tau$ is denoted as $f * g$. The Fourier transform of a function $f(t) \in L^{2}(\mathbb{R})$ is denoted by $\mathcal{F}\{f(t)\} \equiv \hat{f}(j \omega)$, where $L^{2}(\mathbb{R})$ is the function space of all real-valued square-integrable functions. If $\Omega: \mathscr{X} \rightarrow \mathscr{Z}$ is a linear mapping, where $\mathscr{X}$ and $\mathscr{Z}$ are $\mathbb{K}$-vector spaces ( $\mathbb{K}$ a field), the image and the kernel of $\Omega$ are denoted as $\operatorname{Im}\{\Omega\} \subset \mathscr{Z}$ and $\operatorname{Ker}\{\Omega\} \subset \mathscr{X}$, respectively. Finally, the symbol $\varepsilon_{n} \in \mathbb{R}^{n \times 1}$ denotes a vector with odd components equal to 1 and even components equal to 0 .

In the remainder of this section, the formal definitions of two important operators are presented, since their definition in the literature can often be ambiguous.

Definition 1. (Brewer, 1978) (Kronecker sum) The Kronecker sum between two matrices $P_{1}$ and $P_{2}$, with $P_{1} \in \mathbb{R}^{n \times n}$ and $P_{2} \in \mathbb{R}^{k \times k}$, is defined (and denoted) as

$P_{1} \hat{\oplus} P_{2} \triangleq P_{1} \otimes \mathbb{1}_{k}+\mathbb{1}_{n} \otimes P_{2}$.

Definition 2. (Brewer, 1978) (Vec operator) Given a matrix $P=\left[p_{1}, p_{2}, \ldots, p_{m}\right] \in \mathbb{R}^{n \times m}$, where $p_{j} \in \mathbb{R}^{n}, j=1, \ldots, m$, the vector valued operator vec is defined as

$\operatorname{vec}\{P\} \triangleq\left[\begin{array}{c}p_{1} \\ p_{2} \\ \vdots \\ p_{m}\end{array}\right] \in \mathbb{R}^{n m}$.

Finally, useful theorems and properties of the Kronecker sum, and the vec operator, are recalled in the following.

Theorem 1. (Brewer, 1978) Consider matrices $P_{1}$ and $P_{2}$ as in Definition 1. Assume that $P_{1}$ and $P_{2}$ have eigenvalues $\lambda_{i}$, for $i=1, \ldots, n$, and $\mu_{j}$, for $j=1, \ldots, k$. Then the Kronecker sum $P_{1} \oplus \hat{P} P_{2}$ has the nk eigenvalues

$\lambda_{1}+\mu_{1}, \ldots, \lambda_{1}+\mu_{k}, \lambda_{2}+\mu_{1}, \ldots, \lambda_{2}+\mu_{k}, \ldots, \lambda_{n}+\mu_{k}$.

Corollary 1. (Brewer, 1978) The Kronecker sum $P_{1} \oplus \hat{} P_{2}$ is invertible if and only if $\sigma\left(P_{1}\right) \cap \sigma\left(-P_{2}\right)=\varnothing$.

Property 1. (Brewer, 1978) Let $P_{3} \in \mathbb{R}^{n \times m}$ and $P_{4} \in \mathbb{R}^{p \times q}$. The following relation for the vec operator holds:

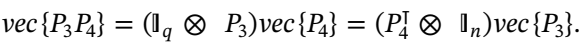

\section{Model order reduction by moment-matching}

To keep this paper reasonably self-contained, several concepts and definitions on moment-matching theory, as formulated in key studies (such as (Astolfi, 2010)), are recalled. Subsequent studies, such as, for example (Scarciotti and Astolfi, 2015), and (Scarciotti and Astolfi, 2016a), exploit this moment characterization to obtain new results regarding the model reduction problem, under diverse assumptions. A brief summary of the key elements of moment-based theory is presented in the following.

\subsection{Moments for linear systems}

In this subsection, the notion of moment for linear systems, as formulated in (Astolfi, 2010), is recalled. Consider a finite-dimensional, single-input, single-output, continuous-time system described, for $t \geq 0$, by the state-space model

$\dot{x}(t)=A x(t)+B u(t)$,

$y(t) \quad=C x(t)$,

where $x(t) \in \mathbb{R}^{n}, \quad u(t) \in \mathbb{R}, \quad y(t) \in \mathbb{R}, \quad A \in \mathbb{R}^{n \times n}, \quad B \in \mathbb{R}^{n \times 1}$ and $C \in \mathbb{R}^{1 \times n}$. Consider the associated transfer function

$W(s)=C\left(s \rrbracket_{n}-A\right)^{-1} B$ and assume that (5) is minimal (i.e controllable and observable).

Definition 3. (Antoulas, 2005) The 0-moment of system (5) at $s_{i} \in \mathbb{C} \backslash \sigma(A)$ is the complex number $\eta_{0}\left(s_{i}\right)=C\left(s_{i} \rrbracket_{n}-A\right)^{-1} B$. The kmoment of system (5) at $s_{i} \in \mathbb{C}$ is the complex number

$\eta_{k}\left(s_{i}\right)=\frac{(-1)^{k}}{k !}\left[\frac{d^{k}}{d s^{k}}\left(C\left(s \|_{n}-A\right)^{-1} B\right)\right]_{s=s_{i}}$,

with $k \geq 1$ integer.

Remark 1. Note that the moments, as in Definition 3, are the coefficients of the Laurent expansion of the transfer function $W(s)$ at the complex numbers $s_{i}$. Model reduction by moment-matching is based on the idea of interpolating the transfer function of the original system (and the derivatives of this) and the transfer function of the reduced order model (and the derivatives of this) at these specific interpolation points $s_{i}$.

In (Astolfi, 2010), it is shown that the moments of system (5) are in a one-to-one relation with the steady-state response (provided it exists) of the output of the interconnection between a signal generator and system (5). This result is recalled, without Proof, in the following theorem (the reader is referred to (Astolfi, 2010; Scarciotti and Astolfi, 2017a) for a comprehensive proof).

Theorem 2. (Astolfi, 2010), (Scarciotti and Astolfi, 2017a), (Scarciotti and Astolfi, 2017b) Consider system (5) and the signal generator

$\dot{\xi}(t)=S \xi(t)$,

$u(t)=L \xi(t)$,

with $\xi(t) \in \mathbb{R}^{\nu \times 1}, S \in \mathbb{R}^{\nu \times \nu}, L \in \mathbb{R}^{1 \times \nu}$ and $\xi(0) \in \mathbb{R}^{\nu \times 1}$. Assume that the triple $(L, S, \xi(0))$ is minimal, $\sigma(A) \subset \mathbb{C}^{-}, \sigma(S) \subset \mathbb{C}^{0}$ and the eigenvalues of $S$ are simple. Let $\Pi \in \mathbb{R}^{n \times v}$ be the (unique) solution of the Sylvester equation

$A \Pi+B L=\Pi S$.

Then, there exists a one-to-one relation between the moments $\eta_{0}\left(s_{1}\right)$, $\eta_{0}\left(s_{2}\right), \ldots, \eta_{0}\left(s_{\nu}\right)$, with $s_{i} \in \sigma(S)$ for all $i=1, \ldots, \nu$, and the steady-state response $C \Pi \xi$ of the output y of the interconnection of system (5) with the signal generator (8) (as in Fig. 1). In fact, the moments are uniquely determined by the matrix $C \Pi$.

Moreover, system (5) has a global invariant manifold described by $\mathcal{M}=\left\{(x, \xi) \in \mathbb{R}^{n+\nu \times 1}: x=\Pi \xi\right\}$. Hence, the expression, $\forall t \geq 0$,

$x(t)=\Pi \xi(t)+e^{A t}(x(0)-\Pi \xi(0))$,

holds.

As discussed in (Scarciotti and Astolfi, 2017b), the assumption on the eigenvalues of $S$ is a sensible hypothesis, since any contribution from a stable mode will decay exponentially to zero. The minimality of the triple $(L, S, \xi(0))$ implies the observability of $(L, S)$ and the controllability of $(S, \xi(0))$.

Remark 2. Note that the steady-state output $y_{s s}$ of the interconnected system in Fig. 1 can be computed from (10) as $y_{S S}(t)=C \Pi e^{S t} \xi(0)$

Remark 3. From now on, the matrix $C \Pi \equiv \bar{Y}$ is referred to as the moment-domain equivalent of $y(t)$.

Finally, the following key result is recalled from (Astolfi, 2010; Scarciotti and Astolfi, 2017a).

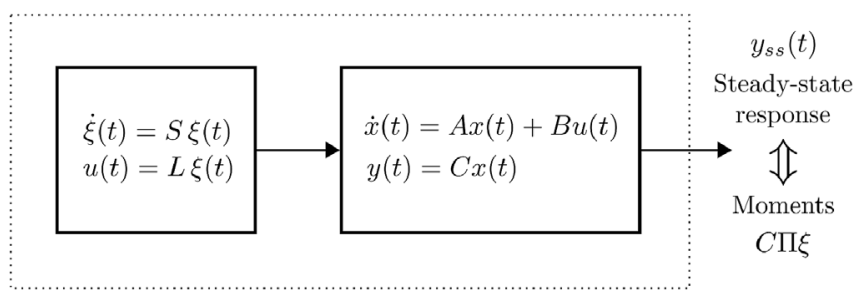

Fig. 1. Schematic of the interconnection between system (5) and the signal generator (8) (adapted from (Astolfi, 2010)). 
Theorem 3. (Astolfi, 2010), (Scarciotti and Astolfi, 2017a) The family of systems described by

$$
\begin{gathered}
\dot{\Theta}(t)=(S-G L) \Theta(t)+G u(t), \\
\theta(t) \quad=\bar{Y} \Theta(t),
\end{gathered}
$$

parametrised on $G \in \mathbb{R}^{v \times 1}$, such that $\sigma(S-G L) \cap \sigma(S) \neq \varnothing$, contains all the models of dimension $\nu$ interpolating the moments of system (5) at $\sigma(S)$.

Remark 4. The transfer function of the reduced order model (11) interpolates the transfer function of system (5) at the frequencies induced by the eigenvalues of S. Equivalently, the steady-state output of the reduced order model (11) exactly matches the steady-state output of the system resulting from the interconnection of system (5) and the signal generator (8).

Remark 5. The matrix G can be selected to enforce specific properties of the original system on the reduced order model, such as a set of prescribed eigenvalues, as detailed in (Astolfi, 2010; Scarciotti and Astolfi, 2017a) and considered in Section 4.1.

\section{WEC equations of motion}

To simplify the notation, a 1-DoF (degree of freedom) WEC is considered in this study, since the extension of the algorithm to multiple degrees of freedom is straightforward. Specifically, taking into account that the steady-state response for each degree of freedom can be readily obtained from hydrodynamic codes, each frequency-response datapoint can be approximated individually, as already exploited in studies such as (Kristiansen et al., 2005) and (Pérez and Fossen, 2008; Perez and Fossen, 2009).

\subsection{Time-domain formulation}

The linearised equation of motion for a 1-DoF device can be expressed in time-domain in terms according to Newton's second law, obtaining the following linear hydrodynamic formulation:

$m \ddot{x}(t)=\mathcal{F}_{r}(t)+\mathcal{F}_{h}(t)+\mathcal{F}_{e}(t)+u(t)$,

where $m$ is the mass of the buoy, $x(t)$ the device excursion, $\mathcal{F}_{e}(t)$ the wave excitation force, $\mathcal{F}_{r}(t)$ the radiation force, $\mathcal{F}_{h}(t)$ the hydrostatic restoring force and $u(t)$ represents a control input, which is supplied by the means of a Power Take-Off (PTO) system. The linearised hydrostatic force for a floating body can be written as $\mathcal{F}_{h}(t)=-s_{h} x(t)$, where $s_{h}>0$ denotes the hydrostatic stiffness. The radiation force $\mathcal{F}_{r}(t)$ is modelled from linear potential theory and, using the well-known Cummins' equation (Cummins, 1962), is

$\mathcal{F}_{r}(t)=-\mu_{\infty} \ddot{x}(t)-\int_{0}^{+\infty} k(\tau) \dot{x}(t-\tau) d \tau$,

where $\mu_{\infty}=\lim _{\omega \rightarrow+\infty} A(\omega), \mu_{\infty}>0$ represents the added-mass at infinite frequency and $k(t) \in L^{2}(\mathbb{R})$ is the (causal) radiation impulse response, containing all the memory effects of the fluid response. Finally, the complete linearised equation of motion of the WEC is given by

$\left(m+\mu_{\infty}\right) \ddot{x}(t)+k(t) * \dot{x}(t)+s_{h} x(t)=\mathcal{F}_{e}(t)+u(t)$,

Equation (14) is of a Volterra integro-differential form, specifically of the convolution class (Wazwaz, 2011). The internal stability of such an equation, for the WEC case, has been analysed and guaranteed for any physically meaningful values of the parameters and the convolution kernel $k(t)$ (Falnes, 2002). In the following, and similarly to the analysis developed in (Ringwood et al.), it is assumed that the PTO input $u(t)$ can be parametrised as

$u(t)=m_{u} \ddot{x}(t)+b_{u} \dot{x}(t)+s_{u} x(t)$,

where the values of $\left\{m_{u}, b_{u}, s_{u}\right\} \in \mathbb{R}$ can be obtained by several optimal (or suboptimal) control strategies (Faedo et al.).

\subsection{Frequency-domain formulation}

As discussed in Section 1, standard hydrodynamic codes provide a frequency-domain response characteristic of the device being analysed, since the direct computation of fluid forces by boundary element methods, or finite volume methods, in the time-domain can be extremely computationally expensive. In the following, and motivated by the popular use of BEM solvers in the literature, the frequency-domain analysis of the WEC dynamics is discussed. Applying the Fourier transform to (14), and considering velocity as the measured output, the following representation

$\hat{\dot{x}}(j \omega)=\hat{\mathcal{F}}_{e}(j \omega) H(j \omega)$,

where $H(j \omega)$ denotes the force-to-velocity frequency response, holds. Note that the expression force-to-velocity (or in the more general case, force-to-motion) is used here to denote the frequency response of the WEC considering excitation force as the input to the system. $H(j \omega)$ is a function of a specific set of frequency-dependent parameters, namely

$H(j \omega)=\frac{1}{b_{u}+B(\omega)+j \omega\left[A(\omega)+m+m_{u}\right]+\frac{s_{h}+s_{u}}{j \omega}}$,

where $B(\omega)$ and $A(\omega)$ represent the radiation damping, and the radiation added mass of the device, respectively (Falnes, 2002). Such parameters can be efficiently obtained using state-of-the-art hydrodynamic solvers, as WAMIT or NEMOH.

Remark 6. Hydrodynamic solvers compute the parameters $B(\omega)$ and $A(\omega)$ for a finite subset of frequency samples $\omega_{i} \in(0, \infty)$. If necessary, different reconstruction procedures could be applied to improve the data obtained (see for example (Jordán and Beltrán-Aguedo, 2004)).

Remark 7. The force-to-position frequency response can be computed from (17) as $P(j \omega)=(j \omega)^{-1} H(j \omega)$.

\subsection{Ogilvie's relations: mapping between time and frequency}

Francis Ogilvie (1964) established a direct relationship between the time-domain (14) and frequency-domain (16) models, as a function of the parameters $B(\omega)$ and $A(\omega)$, and the radiation kernel $k(t)$, using the essential definition of the Fourier transform, namely

$B(\omega) \quad=\int_{0}^{+\infty} k(t) \cos (\omega t) d t$,

$A(\omega)=\mu_{\infty}-\frac{1}{\omega} \int_{0}^{+\infty} k(t) \sin (\omega t) d t$.

It follows that the impulse response $k(t)$ can be written as a mapping involving the frequency-dependent parameters as

$k(t)=\frac{2}{\pi} \int_{0}^{+\infty} B(\omega) \cos (\omega t) d \omega$.

Equation (19) allows a frequency-domain representation of $k(t)$ : a direct application of the Fourier transform, yields

$\hat{k}(j \omega)=B(\omega)+j \omega\left[A(\omega)-\mu_{\infty}\right] \equiv K(j \omega)$.

The radiation kernel frequency response $K(j \omega)$ has a set of particular properties, which have been used in the literature to enforce a structure on the parametric model used to identify the frequency domain data (see, for example (Taghipour et al., 2008), and (Pérez and Fossen, 2008)), in an attempt to improve the quality of the obtained model. In the following, some of these properties are recalled from (Pérez and Fossen, 2008) in Table 1. This is done with the final aim of showing that the reduced order models, obtained by this momentmatching strategy, can inherently respect such properties, since matching the steady-state response of the original system at crucial frequency values, potentially helps to enforce the physical characteristics of the device under analysis.

The reader is referred to (Taghipour et al., 2008) for a 
Table 1

Properties of the radiation force kernel $k$.

\begin{tabular}{ll}
\hline Property & Significance on $\mathrm{k}$ \\
\hline $\lim _{\omega \rightarrow 0} K(j \omega)=0$ & It has zeros at the origin \\
$\lim _{\omega \rightarrow+\infty} K(j \omega)=0$ & Strictly proper \\
$\lim _{t \rightarrow+\infty} k(t)=0$ & BIBO stable \\
$\mathfrak{R}\{K(j \omega)\} \geq 0$ & Passive $^{1}$ \\
\hline
\end{tabular}

comprehensive demonstration of each property listed in Table 1.

\section{Moment-based WEC formulation}

The development of model order reduction by moment-matching theory, as described in Section 2.1, is based on a state-space representation of the system being approximated. Therefore, the equation of motion presented in (14) needs to be re-written in a more suitable structure. The following state-space representation, for the WEC dynamics, is proposed:

$\dot{\varphi}(t)=A_{\varphi} \varphi(t)+B_{\varphi} \mathfrak{u}(t)$,

$y_{\varphi}(t) \quad=C_{\varphi} \varphi(t)$,

where $\varphi(t)=[x(t), \dot{x}(t)]^{\top} \in \mathbb{R}^{n \times 1}$, with $n=2$, is the state-vector of the continuous-time model and $y_{\varphi}(t)=\dot{x}(t) \in \mathbb{R}$ is the output of the system (assuming velocity as the measurable output of the device). The function $u(t) \in \mathbb{R}$, assumed to be the input of system (21), is defined as

$\mathfrak{u}(t)=\mathcal{F}_{e}(t)-k(t) * \dot{x}(t)$,

Under this assumption, the matrices in (21) are given by

$A_{\varphi}=\left[\begin{array}{cc}0 & 1 \\ -\frac{s h+s_{u}}{m+\mu_{\infty}+m_{u}} & -\frac{b_{u}}{m+\mu_{\infty}+m_{u}}\end{array}\right], \quad B_{\varphi}=\left[\begin{array}{c}0 \\ \frac{1}{m+\mu_{\infty}+m_{u}}\end{array}\right]$,

$C_{\varphi}=\left[\begin{array}{ll}0 & 1\end{array}\right]$.

Remark 8. The radiation force convolution term is included as a feedback term in $\mathfrak{u}(t)$, only as an algebraic manipulation to develop a state-space representation of (14). Note that the meaningful input is the wave excitation force $\mathcal{F}_{e}(t)$.

Within the moment-based framework, the input $\mathcal{F}_{e}$ is expressed as a signal generator (8), written in implicit form as

$\dot{\xi}_{e}(t)=S \xi_{e}(t)$,

$\mathcal{F}_{e}(t)=L_{e} \xi_{e}(t)$

where the dimension of $S$ and $L$ are as in (8), $\xi_{e}(t) \in \mathbb{R}^{v \times 1}$ and, without loss of generality, the initial condition of the signal generator is chosen as $\xi_{e}(0)=\varepsilon_{\nu}$. Since the eigenvalues of $S$ are simple and lie in $\mathbb{C}^{0}, S$ can be written in a real block-diagonal form as
$S=\underset{p=1}{f}\left[\begin{array}{cc}0 & \omega_{p} \\ -\omega_{p} & 0\end{array}\right]$

where $v=2 f, f \geq 0$ integer. Note that with this selection of matrices, the assumption on the minimality of the triple $\left(L, S, \xi_{e}(0)\right)$ holds as long as the pair $(L, S)$ is observable. Also note that each $\omega_{p}$ represents a desired interpolation point for the model reduction process (see Remark 4), i.e. a frequency where the transfer function of the reduced order model matches the transfer function of the original system.

Remark 9. Note that the specific structure of $S$ described in Equation (25) implies that the excitation force is described as the sum of several frequency components, defined by the spectrum of the matrix S. For example, if $S=\left[\begin{array}{cc}0 & \gamma \\ -\gamma & 0\end{array}\right]$ and $L=\left[\begin{array}{ll}\alpha_{1}-\alpha_{2}\end{array}\right]$ then $\mathcal{F}_{e}(t)=\alpha_{1} \cos (\gamma t)+\alpha_{2} \sin (\gamma t)$ with $\left\{\gamma, \alpha_{1}, \alpha_{2}\right\} \in \mathbb{R}$.

Under this selection of matrices, the moments of system (21), driven by the signal generator (24) (as in Fig. 2), can be computed by solving a Sylvester equation (see Theorem 2). Considering superposition, the Sylvester equation for the WEC device case can be written as

$A_{\varphi} \Pi_{\varphi}+B_{\varphi}\left(L_{e}-\bar{Z}\right)=\Pi_{\varphi} S$,

where $\Pi_{\varphi} \in \mathbb{R}^{n \times v}$ and $\bar{Z}$ is the moment-domain equivalent of the radiation convolution term. Note that the moment-domain equivalent of the velocity can simply be expressed in terms of the solution of (26) as $\bar{V}=C_{\varphi} \Pi_{\varphi}$.

Remark 10. (On the uncontrolled case). One of the main assumptions of Theorem 2 is that the system being analysed is internally stable, i.e. $\sigma(A) \subset \mathbb{C}^{-}$in (9). Such an assumption guarantees the existence of the steady-response of the interconnected system, so that the equivalence between moments and steady-state response holds. Although that, if $u(t)=0$ (uncontrolled WEC) in (21) then $\sigma\left(A_{\varphi}\right) \subset \mathbb{C}^{0}$, the steady-state response of system (21) is well-defined, due to the internal stability of (14) (Falnes, 2002).

Despite the fact that an analytical expression has been derived for $\bar{Z}$ in (Faedo et al. ), a Proof is provided in this study, for convenience.

Proposition 1. (Faedo et al. ) The moment-domain equivalent of the convolution integral in (13) can be computed as

$\bar{Z}=\bar{V} \mathcal{R}$,

where $\mathcal{R} \in \mathbb{R}^{\nu \times v}$ is a block-diagonal matrix defined by

$\mathcal{R}=\bigoplus_{p=1}^{f}\left[\begin{array}{cc}r_{\omega_{p}} & -m_{\omega_{p}} \\ m_{\omega_{p}} & r_{\omega_{p}}\end{array}\right]$,

and its entries depend on the added mass $A(\omega)$ and the radiation damping $B(\omega)$ of the device at each specific frequency induced by the eigenvalues of $S$, as

$r_{\omega_{p}}=\mathcal{B}\left(\omega_{p}\right), m_{\omega_{p}}=-\omega_{p}\left[\mathscr{A}\left(\omega_{p}\right)-\mu_{\infty}\right]$.

Proof.

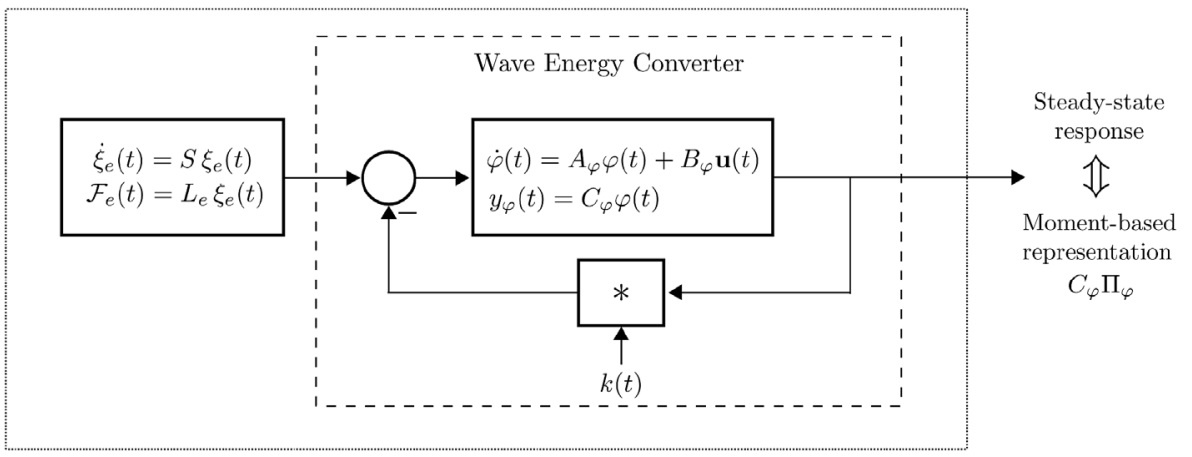

Fig. 2. Block-diagram of the interconnection between system (21) and the signal generator (24). 
Recalling Remark 2, the steady-state response of the convolution integral can be computed in the moment-domain as

$\int_{0}^{+\infty} k(\tau) \dot{x}(t-\tau) d \tau=\bar{V} \int_{0}^{+\infty} k(\tau)\left(e^{S(t-\tau)} \varepsilon_{\nu}\right) d \tau$.

Note that that the vector $e^{S t} \varepsilon_{v} \in \mathbb{R}^{v \times 1}$ can be conveniently expanded as

$e^{S t} \varepsilon_{v}=\left[\begin{array}{c}\cos \left(\omega_{1} t\right) \\ -\sin \left(\omega_{1} t\right) \\ \vdots \\ \cos \left(\omega_{k} t\right) \\ -\sin \left(\omega_{k} t\right)\end{array}\right]=\left[\begin{array}{c}\psi_{\omega_{1}}^{+}(t) \\ \psi_{\omega_{1}}^{-}(t) \\ \vdots \\ \psi_{\omega_{k}}^{+}(t) \\ \psi_{\omega_{k}}^{-}(t)\end{array}\right]$,

so that the convolution integral (30) can be written in a more suitable (vector) form, namely

$\bar{V}\left[\begin{array}{c}k(t) * \psi_{\omega_{1}}^{+}(t) \\ k(t) * \psi_{\omega_{1}}^{-}(t) \\ \vdots \\ k(t) * \psi_{\omega_{k}}^{+}(t) \\ k(t) * \psi_{\omega_{k}}^{-}(t)\end{array}\right]$.

Taking elements $\psi_{\omega_{p}}^{+}(t), \psi_{\omega_{p}}^{-}(t)$, it is possible to recognize two general convolution operations, i.e. $k(t) * \psi_{\omega_{p}}^{+}(t)$ and $k(t) * \psi_{\omega_{p}}^{-}(t)$. Expanding the first expression, and considering well-known trigonometric identities, yields

$$
\begin{gathered}
k(t) * \psi_{\omega_{p}}^{+}(t)=\cos \left(\omega_{p} t\right) \int_{0}^{+\infty} k(t) \cos \\
\left(\omega_{p} t\right) d t+ \\
\sin \left(\omega_{p} t\right) \int_{0}^{+\infty} k(t) \sin \\
\left(\omega_{p} t\right) d t .
\end{gathered}
$$

Using Oglivie's relationships, defined in (18), the integral operations involved in (33) can be evaluated explicitly, using the frequency-dependent parameters $B(\omega)$ and $A(\omega)$ :

$$
\begin{gathered}
\int_{0}^{+\infty} k(t) \cos \left(\omega_{p} t\right) d t \quad=B\left(\omega_{p}\right)=r_{\omega_{p}}, \\
\int_{0}^{+\infty} k(t) \sin \left(\omega_{p} t\right) d t=-\omega_{p}\left[A\left(\omega_{p}\right)-\mu_{\infty}\right]=m_{\omega_{p}},
\end{gathered}
$$

Performing similar operations on $k(t) \backslash$ scalebox $1.5 * \psi_{\omega_{p}}^{-}(t)$ the expression

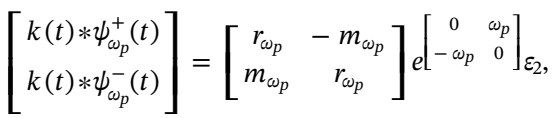

holds. Finally, considering the totality of the convolution operations in (32), the Proof follows.

With the analytical definition of the moment-domain equivalent of the radiation force convolution term in (27), the following proposition is made, to compute the solution of the Sylvester equation (26).

Proposition 2. The moment-domain equivalent of the output $y_{\varphi}$ of system (21) can be computed as

$\bar{V}=L_{e} \Phi_{\varphi}^{\mathcal{R}}$,

where

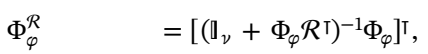

$\Phi_{\varphi}=\left(\mathbb{I}_{\nu} \otimes C_{\varphi}\right)\left(S \oplus A_{\varphi}\right)^{-1}\left(\mathbb{I}_{\nu} \otimes-B_{\varphi}\right)$,

with $\Phi_{\varphi}^{\mathcal{R}} \in \mathbb{R}^{\nu \times \nu}$ and $\Phi_{\varphi} \in \mathbb{R}^{n \nu \times n \nu}$.

Proof.

Equation (26) can be analysed using a linear geometric approach. For convenience, (26) is re-written as

$A_{\varphi} \Pi_{\varphi}+\Pi_{\varphi}(-S)=-B_{\varphi}\left(L_{e}-\bar{Z}\right)$.
From a geometric perspective, (38) can be seen as a linear endomorphism $\Phi$ over $\mathbb{R}^{n \times v}$, i.e.

$$
\begin{gathered}
\Phi: \mathbb{R}^{n \times \nu} \rightarrow \mathbb{R}^{n \times \nu}, \quad \Pi_{\varphi} \in \mathbb{R}^{n \times \nu}, \\
\Phi\left\{\Pi_{\varphi}\right\} \mapsto A_{\varphi} \Pi_{\varphi}+\Pi_{\varphi}(-S),
\end{gathered}
$$

and then the matrix $-B_{\varphi}\left(L_{e}-\bar{Z}\right) \in \operatorname{Im}\{\Phi\} \subset \mathbb{R}^{n \times \nu}$. Considering an ordered canonical basis for $\mathbb{R}^{n \times v}$, in accordance with the vec operator (see Definition 2), the elements of $\Pi_{\varphi}$ in (39) can be computed as ${ }^{1}$ (Van Loan, 2000)

$\operatorname{vec}\left\{\Pi_{\varphi}\right\}=\left(S \hat{\oplus} A_{\varphi}\right)^{-1} \operatorname{vec}\left\{-B_{\varphi}\left(L_{e}-\bar{Z}\right)\right\}$,

where the existence of $\left(S \oplus^{\wedge} A_{\varphi}\right)^{-1}$ is guaranteed by Corollary 1 , since $\sigma\left(A_{\varphi}\right) \cap \sigma(S)=\varnothing$ for any realistic device parameters involved in the matrix $A_{\varphi}$ and, therefore, $\Phi$ is an automorphism, i.e. $\operatorname{Ker}\{\Phi\}=\{0\}$. Using the vec operator equivalence stated in Property 1 , and recalling that $\bar{V}=C_{\varphi} \Pi_{\varphi}$, the following equality,

$\operatorname{vec}\{\bar{V}\}=\Phi_{\varphi}\left(\operatorname{vec}\left\{L_{e}\right\}-\operatorname{vec}\{\bar{Z}\}\right)$,

holds. Substituting the moment-domain equivalent of the radiation convolution term obtained in (27) and, after algebraic manipulations, the expression obtained in (41) can be written as

$\operatorname{vec}\{\bar{V}\}=\left[\left(\mathbb{I}_{\nu}+\Phi_{\varphi} \mathcal{R}\right)^{-1} \Phi_{\varphi}\right] \operatorname{vec}\left\{L_{e}\right\}$.

Finally, by recalling that the basis considered for the computation of (40) is canonical, the coordinates of the moment-domain equivalents, and the moment-domain equivalents themselves, are related by a simple transposition operation, i.e. $\operatorname{vec}\{\bar{V}\}=\bar{V}^{\top}$, $\operatorname{vec}\left\{L_{e}\right\}=L_{e}^{\top}$, and the Proof follows. .

Proposition 2 shows an explicit analytical expression for the momentdomain equivalent of the output of system (21). Such a result allows the computation of a reduced order model of system (21) using Theorem 3 in a straightforward way. Explicitly:

$\widetilde{\mathcal{H}}_{\sigma(S)}:\left\{\begin{array}{c}\dot{\Theta}_{\varphi}(t)=\left(S-G_{\varphi} L_{e}\right) \Theta_{\varphi}(t)+G_{\varphi} \mathcal{F}_{e}(t), \\ \theta_{\varphi}(t) \quad=\bar{V} \Theta_{\varphi}(t),\end{array}\right.$

is the family of reduced order models, parametrised in $G_{\varphi}$, interpolating the moments of system (21) at the eigenvalues of $S$, where $\bar{V}=L_{e} \Phi_{\varphi}^{\mathcal{R}}$.

Remark 11. The reduced order model (43) has dimension $v=2 f$, where $f$ is the number of interpolation points (frequencies) selected. This is a consequence of the fact that, for each frequency $\omega_{i}$, both $\pm j \omega_{i}$ are chosen as eigenvalues of the real-valued matrix $\mathrm{S}$.

Remark 12. The notation $\widetilde{\mathcal{H}}_{\sigma(S)}$ refers to an approximated time-domain model of the force-to-velocity dynamics of the device under analysis, by matching the frequencies selected in $\sigma(S)$.

\subsection{Eigenvalue assignment}

As discussed in Remark 5, the additional degree of freedom provided by $G_{\varphi}$ can be exploited to arbitrarily assign the eigenvalues of the reduced order model (43), i.e. given a set of eigenvalues $\Sigma_{\varphi}$, one can select $G_{\varphi}$ such as $\sigma\left(S-G_{\varphi} L_{e}\right)=\Sigma_{\varphi}$. Note that $G_{\varphi}$ is guaranteed to be unique, due to the observability of the pair $\left(L_{e}, S\right)$. In this particular case, the set of desired eigenvalues is chosen within an optimisation formulation, which attempts to minimise the euclidean distance between the device frequency response $H(j \omega)$, constructed with data obtained with hydrodynamic codes (17), and the reduced order model frequency response $\widetilde{H}(j \omega)$, computed from the following transfer function (notation adopted from Remark 12):

$\widetilde{H}_{\sigma(S)}(s)=\bar{V}\left[s \rrbracket_{\nu}-\left(S-G_{\varphi} L_{e}\right)\right]^{-1} G_{\varphi}$.

As already stated in Remark 6, the frequency-dependent device

\footnotetext{
${ }^{1}$ Note also that $S$ is skew-symmetric.
} 
parameters are calculated using hydrodynamic codes at a finite number of frequencies $\omega_{i} \in\left[\omega_{l}, \omega_{u}\right]$, with a frequency step of $\Delta \omega_{i}$, where $\omega_{l}$ and $\omega_{u}$ represents the lower and upper bound of the range, respectively. Such a frequency range depends explicitly on the application (further discussed in Section 5). Define the complex-valued vectors $H_{\omega}, \widetilde{H}_{\omega}$ as,

$$
H_{\omega}=\left[\begin{array}{c}
H\left(j \omega_{l}\right) \\
H\left(j\left(\omega_{l}+\Delta \omega_{i}\right)\right) \\
\vdots \\
H\left(j\left(\omega_{u}\right)\right)
\end{array}\right], \widetilde{H}_{\omega}=\left[\begin{array}{c}
\widetilde{H}_{\sigma(S)}\left(j \omega_{l}\right) \\
\widetilde{H}_{\sigma(S)}\left(j\left(\omega_{l}+\Delta \omega_{i}\right)\right) \\
\vdots \\
\widetilde{H}_{\sigma(S)}\left(j\left(\omega_{u}\right)\right)
\end{array}\right] .
$$

Then, the proposed optimisation procedure, to assign the eigenvalues of the reduced order model $\Sigma_{\varphi} \subset \mathbb{C}^{-}$, can be formulated as,

$\min _{\Sigma_{\varphi} \subset \mathbb{C}^{-}}\left\|H_{\omega}-\widetilde{H}_{\omega}\right\|_{2}^{2}$.

\subsection{Radiation force convolution approximation}

The radiation convolution term in (13) defines a linear time-invariant system completely characterised by the impulse response function $k(t)$, where the input considered is the velocity of the device $\dot{x}(t)$, i.e.

$y_{k}(t)=k(t) * \dot{x}(t)$.

A reduced order model by moment-matching can be obtained using the result on the moment-domain equivalent of such a convolution term, provided in Proposition 1, as developed in the following.

Assume that the velocity $\dot{x}(t)$ of the WEC can be written as a signal generator in implicit form, in a similar fashion to (24), expressed as a set of linear differential equations given by

$\dot{\xi}_{k}(t)=S \xi_{k}(t)$,

$\dot{x}(t)=L_{k} \xi_{k}(t)$,

with $\xi_{k}(0)=\varepsilon_{v}$ and $L_{k}$ such as the pair $\left(L_{k}, S\right)$ is observable. Then, recalling Proposition 1, the moment-domain equivalent of the output of (47) can be straightforwardly computed as $\bar{Y}_{k}=L_{k} \mathcal{R}$, and a reduced order model of (47) can be obtained by applying Theorem 3 . Specifically:

$\widetilde{\mathscr{K}}_{\sigma(S)}:\left\{\begin{array}{c}\dot{\Theta}_{k}(t)=\left(S-G_{k} L_{k}\right) \Theta_{k}(t)+G_{k} \dot{x}(t), \\ \theta_{k}(t) \quad=\bar{Y}_{k} \Theta_{k}(t),\end{array}\right.$

is the family of reduced order models parametrised in $G_{k}$, interpolating the moments of system (47) at the eigenvalues of $S$, where $\overline{Y_{k}}=L_{k} \mathcal{R}$. Following Equation (44), the transfer function of the reduced order model (47) can be computed as

$\widetilde{K}_{\sigma(S)}(s)=\bar{Y}_{k}\left[s \rrbracket_{v}-\left(S-G_{k} L_{k}\right)\right]^{-1} G_{k}$,

and the complex-valued vectors $K_{\omega}$ and $\widetilde{K}_{\omega}$ are defined as in (45), by considering the frequency response of the radiation convolution kernel $K(j \omega)(20)$ (instead of $H(j \omega)$ ), and the reduced order model transfer function $\widetilde{K}_{\sigma(S)}(s)$ (instead of $\widetilde{H}_{\sigma(S)}(s)$ ). Then, the set of desired eigenvalues $\Sigma_{k}$ of system (49), can be assigned using the same optimisation criterion described in Section 4.1, namely,

$\min _{\Sigma_{k} \subset \mathbb{C}^{-}}\left\|K_{\omega}-\widetilde{K}_{\omega}\right\|_{2}^{2}$.

\subsection{Force-to-position dynamic model}

Note that Section 4, describes the theoretical framework to compute a parametric form for the force-to-velocity dynamics (17) and the radiation convolution operation (20), using the moment-matching technique. If a force-to-position parametric form is required, one could either change the vector $C_{\varphi}$ in (21) accordingly, or consider the following

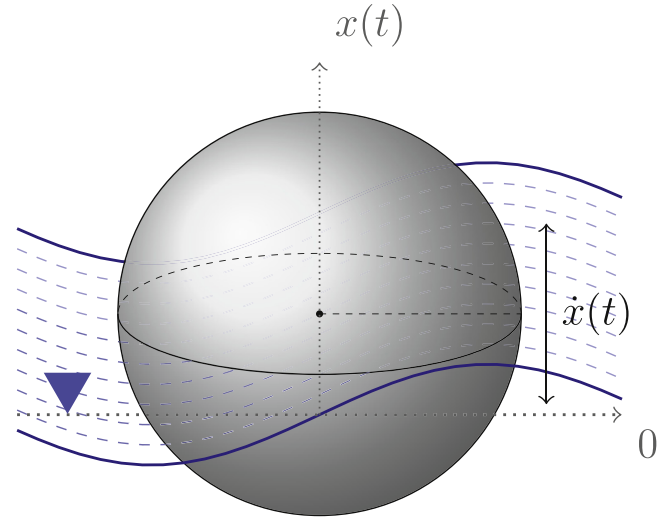

Fig. 3. Heaving point absorber wave energy converter, as an application example.

procedure, which further exploits the properties of the moment-based formulation.

Proposition 3. (Scarciotti and Astolfi, 2016b)Consider a dynamical system given by the differential equation

$\dot{x}(t)=u(t)$.

Then, the moment-domain equivalent of $\dot{x}(t)$ is $X S$, where $X$ is the moment-domain equivalent of $x(t)$. In an analogous form, the momentdomain equivalent of $\int x(\tau) d \tau$ is given by $X S^{-1}$.

Proposition 3allows a parametric form of the force-to-position frequency response $P(j \omega)$ (see Remark 7) to be obtained, using the same results computed for the force-to-velocity response $H(j \omega)$. Specifically, the momentdomain equivalent of the position of the WEC, $\bar{X}$, can be computed as,

$\bar{X}=\bar{V} S^{-1}$,

with $\bar{V}$ as in (36). Hence, the family of reduced order models interpolating $P(j \omega)$ at the eigenvalues of $S$, can be obtained from (43) by simply replacing $\bar{V}$ with $\bar{X}$.

\section{Numerical example}

To present and illustrate the application of this model order reduction method, a $5[\mathrm{~m}]$ diameter spherical heaving point absorber WEC is considered in Section 5.1 and Section 5.2, as illustrated in Fig. 3, while a more geometrically complex device is considered in Section 5.3: the CETO wave energy converter (the reader is referred to (Penalba et al., 2017b) for a discussion on the frequency-domain characteristics of such a device). Though multiple degrees of freedom can be considered, for simplicity, only the vertical motion (heave) is considered for the spherical WEC case, while the surge motion of the CETO device is the focus, in the numerical examples. In both cases, the hydrodynamic coefficients are computed using the BEM solver NEMOH. ${ }^{2}$ (Babarit and Delhommeau, 2015). Note that it is automatically assumed that, in Sections 5.1 and 5.2, the terms "WEC" and "device" are used to denote the spherical WEC depicted in Fig. 3 while, in Section 5.3, these terms are used to denote the CETO converter. Without any loss of generality, it is assumed in the following examples that $m_{u}=b_{u}=s_{u}=0$ i.e. there is no presence of a control input.

The added mass and radiation damping of the spherical WEC can be seen in Fig. 4a, along with the radiation kernel frequency response $K(j \omega)$ in Fig. 4b, and the force-to-velocity frequency response $H(j \omega)$ in Fig. 4c. For this particular example, the maximum frequency selected in the hydrodynamic code, to compute both $A(\omega)$ and $B(\omega)$, is 10 [rad/s]. Nevertheless, ocean waves peak periods typically lie between 3 and

\footnotetext{
${ }^{2}$ A mesh convergence study has been performed for these numerical examples.
} 
a)
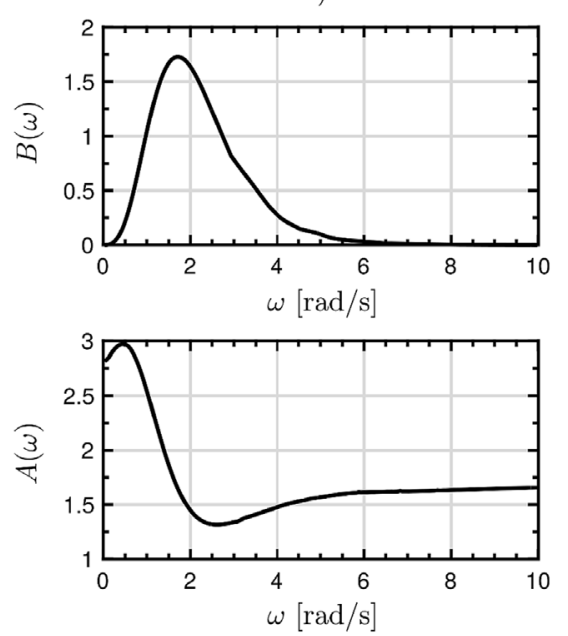

b)
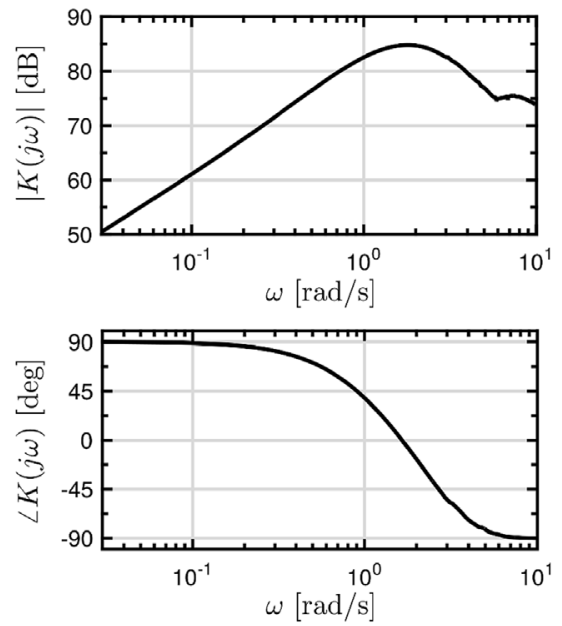

c)
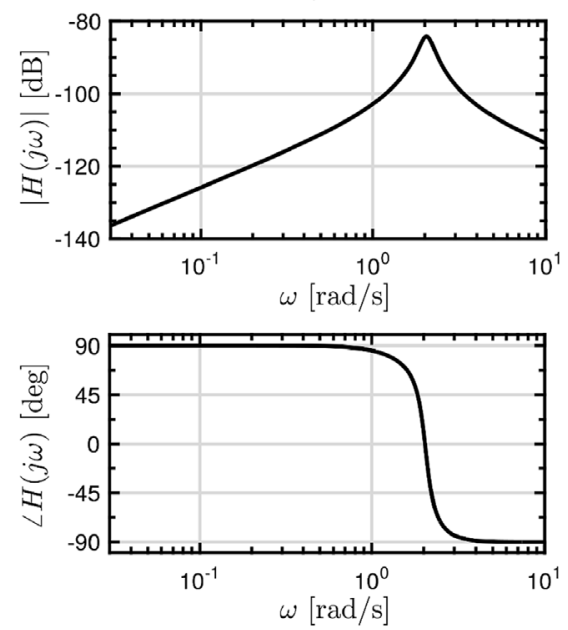

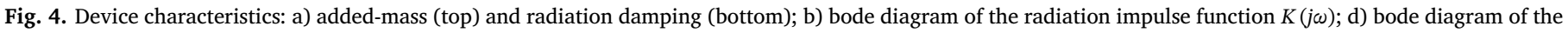
force-to-velocity frequency response $H(j \omega)$.

$16 \mathrm{~s}$, which implies that the frequency range of the excitation force (input of the WEC system) is approximately [0.3, 2.1] [ rad/s]. This phenomenon is consistent across different geographical locations, as discussed in (Penalba et al., 2017b).

For the numerical examples in this section, both regular and irregular waves inputs are studied. In particular, a JONSWAP spectrum (Hasselmann, 1973) is considered for the irregular waves case (Fig. 5), with a peak period of $T_{p}=10$ [s], significant wave height $H_{s}=2[\mathrm{~m}]$ and peak enhancement factor $\gamma=3.3$. Note that a multimodal wave spectrum, such as the Ochi-Hubble spectrum (Ochi and Hubble, 1976), could also be considered in this strategy, though a unimodal wave characteristic is considered in this study to illustrate the numerical results, for simplicity. Considering the spectrum depicted in Fig. 5, the frequency range to approximate the parametric models is selected as $\omega_{l}=0.3[\mathrm{rad} / \mathrm{s}]$ and $\omega_{u}=3[\mathrm{rad} / \mathrm{s}]$.

\subsection{Force-to-velocity parametric model}

In this subsection, the complete force-to-velocity frequency response $H(j \omega)$ is considered for the application of the model order reduction by moment-matching procedure, with wave excitation force $\mathcal{F}_{e}$ as the model input. Recall that the first step of the formulation is to select the frequencies to interpolate, by choosing the eigenvalues of the matrix $S$ in (25), so that a reduced order model can be constructed from (44). Fig. 4c provides a sensible and intuitive way to decide on a set of frequencies to achieve moment-matching, by analysing the frequency response $H(j \omega)$. For example, it is straightforward to notice that the resonant frequency of the device $\omega \approx 2[\mathrm{rad} / \mathrm{s}]$ represents an interpolation point of paramount importance. Note that, if $\omega=2[\mathrm{rad} / \mathrm{s}]$ is selected as interpolation point, the frequency response of the reduced order system will match exactly (up to any numerical imprecision when computing the moments) the behaviour of the device at the resonant frequency.

In the following example, and to illustrate the previously discussed fact on selecting important frequencies, a specific interpolation point is chosen at the resonant frequency of the target system. The Bode diagram of the force-to-velocity frequency response $H(j \omega)$ of the device under analysis, and the reduced order model frequency response $\widetilde{H}_{\{2\}}(j \omega)$, are compared in Fig. 6.

The black dot represents the frequency chosen for the interpolation process (approximate resonant frequency $\omega=2$ ). It can be appreciated that $\widetilde{H}_{\{2\}}(j \omega)$ exactly matches the steady-state behaviour of the device at that particular point, as expected from the theoretical framework of the strategy. Note that the assignment of the eigenvalues of the reduced

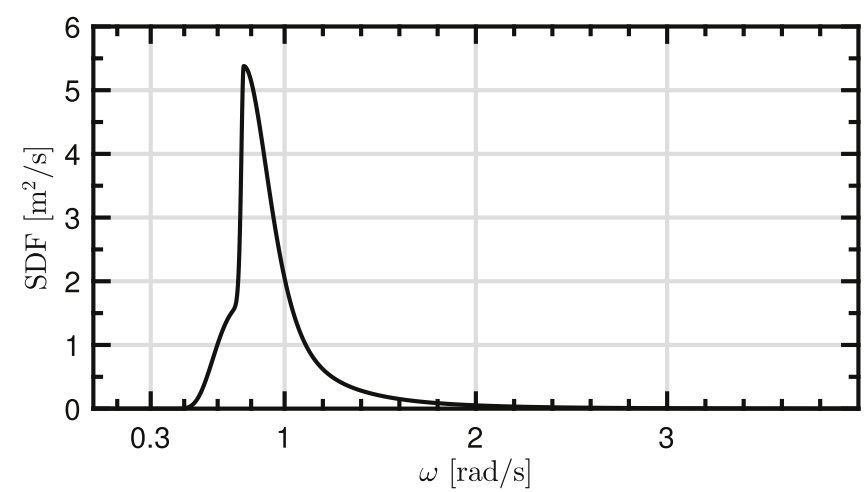

Fig. 5. JONSWAP spectrum considered for the numerical simulation of the obtained parametric models under irregular waves excitation. The white area refers to the frequency range selected to compute an (optimal) approximated parametric model is $[0.3,3]$ [ rad/s].

order model is performed by minimising the difference between the original non-parametric frequency response of and the frequency response of the reduced order model (see Equation (46)), in the area of interest chosen (white area in Fig. 6). Note that the frequency range can be chosen arbitrarily, but would normally represent the operational frequency range of the WEC, as determined by its own dynamics and the incident sea state.

The approximation obtained with $H_{\{2\}}(j \omega)$ (or equivalently, $\widetilde{\mathcal{H}}_{\{2\}}$ in the time-domain) can be further improved by selecting a higher number of interpolation points, but with a consequent increase in model order. For illustration, the reduced order model is re-computed using two interpolation points, namely, the resonant frequency of the device $\omega=2$ and a low frequency component $\omega=0.4$. The magnitude and phase of the model, $\widetilde{H}_{\{2,0.4\}}(j \omega)$, is shown in Fig. 7. If the resulting reduced model is still unsatisfactory, the process of adding interpolation points can be repeated until the approximation obtained is adequate. For this current example, little improvement is observed when considering more than two frequencies in the interpolation scheme. This is further discussed in Section 5.2, where the approximation error is shown to be monotonically decreasing with increasing model order $(\nu)$.

Fig. 8 depicts the time-domain response of the reduced order model $\hat{\mathcal{H}}_{\{2,0.4\}}$ for a regular wave input, with a frequency of $1.4[\mathrm{rad} / \mathrm{s}]$, while Fig. 9 depicts the time-domain response of the same system for an irregular sea state (JONSWAP spectrum described in Fig. 5). It can be 

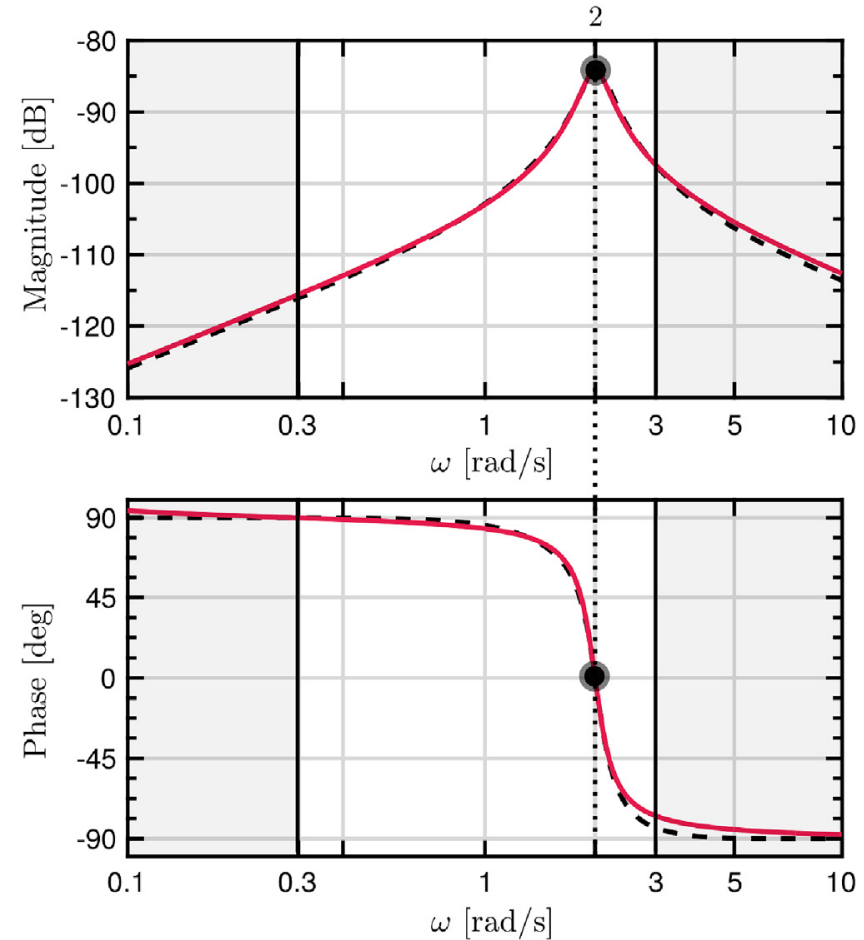

Fig. 6. Bode diagram of the force-to-velocity frequency response for the device computed with NEMOH (dashed-black) and the reduced order model (solid-red) frequency response, considering one interpolation point (specified on top of figure). The white area indicates the frequency range considered for the approximation. (For interpretation of the references to colour in this figure legend, the reader is referred to the Web version of this article.)
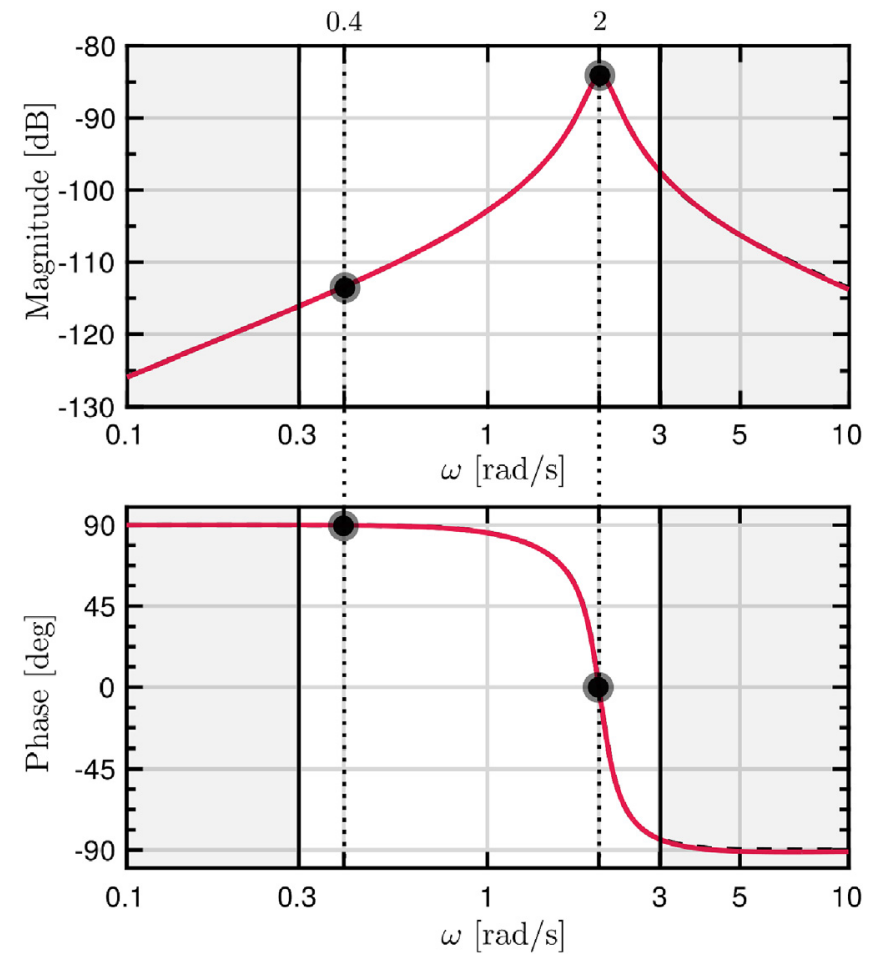

Fig. 7. Bode diagram of the force-to-velocity frequency response for the device computed with NEMOH (dashed-black) and the reduced order model (solid-red) frequency response, considering two interpolation points (specified on top of figure). The white area indicates the region of frequencies considered for the approximation. (For interpretation of the references to colour in this figure legend, the reader is referred to the Web version of this article.)

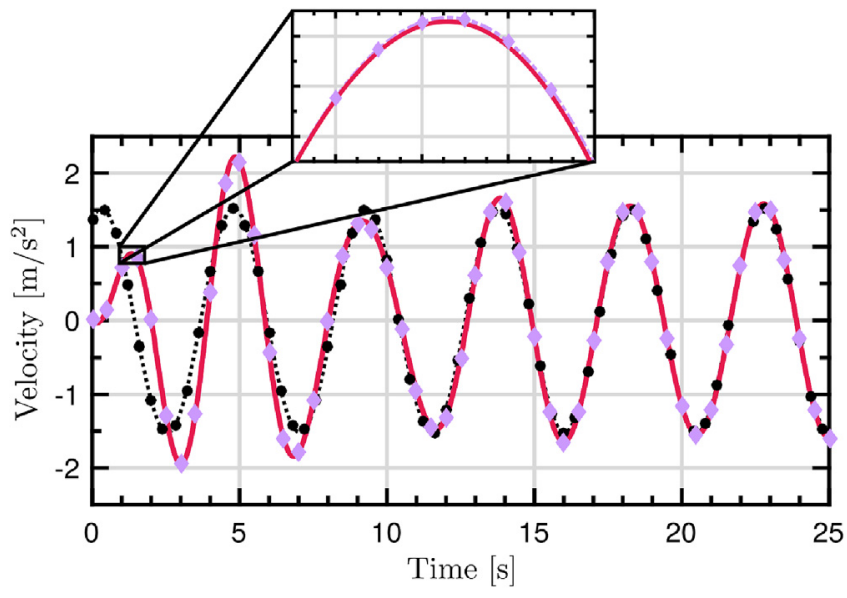

Fig. 8. Comparison between the time-domain output of the force-to-velocity reduced order model using two interpolation points (solid-red), the time-domain response obtained from Cummins' equation computing the convolution product directly (diamond-violet) and the steady-state response computed from the force-to-velocity frequency response of the device under analysis (dottedblack), for an input frequency of $\omega=1.4[\mathrm{rad} / \mathrm{s}]$. (For interpretation of the references to colour in this figure legend, the reader is referred to the Web version of this article.)

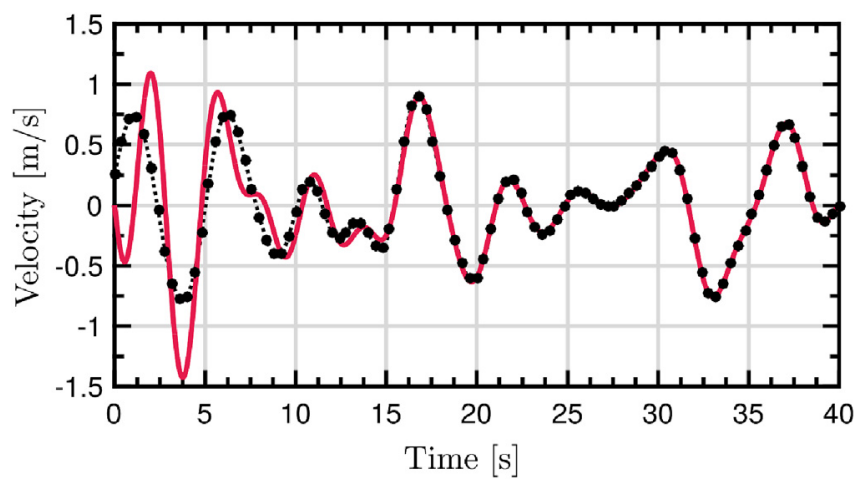

Fig. 9. Comparison between the time-domain output of the force-to-velocity reduced order model using two interpolation points (solid-red) and the steadystate response computed from the force-to-velocity frequency response of the device under analysis (dotted-black), for an irregular sea state. (For interpretation of the references to colour in this figure legend, the reader is referred to the Web version of this article.)

readily seen that the steady-state behaviour of the reduced system remains close to the target steady-state output, under both regular and irregular periodic excitation. Additionally, in Fig. 8, the transient response of the obtained reduced model is compared to the response obtained from Cummins' equation (14) by computing the convolution product directly, and it can be immediately appreciated that both responses are virtually identical. Note that the target steady-state output is computed using the frequency-domain data computed by $\mathrm{NEMOH}$, i.e. the time-domain input is modified in amplitude and phase accordingly to the excitation frequency. Finally, considering the momentmatching framework property described in Section 4.3, a reduced order model, with both velocity and position as outputs, can be obtained straightforwardly, using (53). The phase-plane for a parametric model achieving moment-matching at two frequencies ( 2 and $0.4[\mathrm{rad} / \mathrm{s}]$ ), is shown in Fig. 10, along with the phase-plane of the steady-state response of the device under analysis, for an input frequency of $2[\mathrm{rad} / \mathrm{s}]$.

\subsection{Radiation impulse response parametric model}

The moment-matching framework is now applied to the frequency 


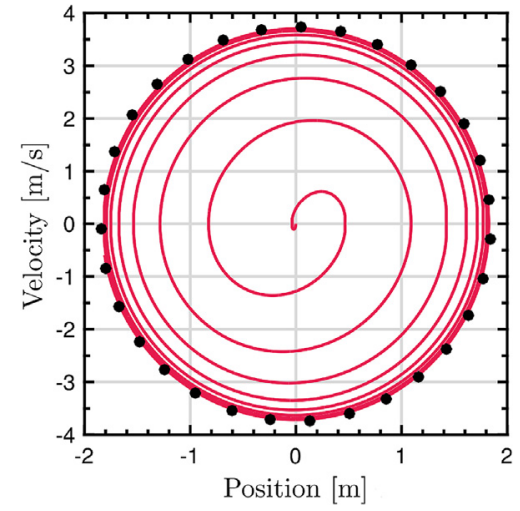

Fig. 10. Phase-plane of the time-domain trajectories of the reduced order model (interpolating two frequencies) (solid-red), and the target steady-state response (dotted-black). (For interpretation of the references to colour in this figure legend, the reader is referred to the Web version of this article.)

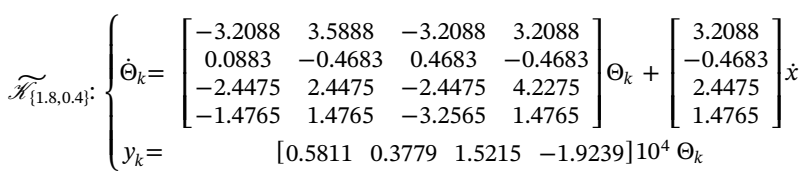

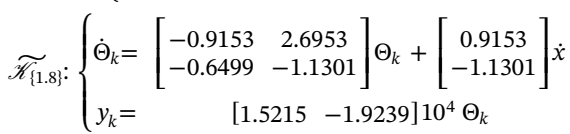

response of the radiation kernel $K(j \omega)$, as developed in Section 4.2. In this case, and after selecting the appropriate interpolation points, the reduced order model by moment-matching is constructed from (49).

Following an analogous procedure to that described in Section 5.1, the interpolation points can be selected by inspecting Fig. 4b. For this case, and considering only one interpolation point, the frequency response of a reduced order model achieving moment-matching at $\omega=1.8, \widetilde{K}_{\{1.8\}}(j \omega)$, is shown in the Bode diagram of Fig. 11 (note that 1.8 $[\mathrm{rad} / \mathrm{s}]$ represents, approximately, an inflection point in the frequency response, analogous to the resonant frequency of the device for the force-to-velocity case discussed in Section 5.1). As in the case of the force-to-velocity dynamics, it is clear that the reduced order model perfectly interpolates the target frequency response at the selected frequency. Again, if the reduced model obtained is unsatisfactory, a higher number of interpolation points can be chosen, as already exploited in the approximation of the force-to-velocity dynamics. Fig. 12 depicts the Bode diagram of both the target frequency response, and the frequency response of a reduced order model obtained by interpolating at $\omega=1.8$ and $\omega=0.4$ (low frequency component). The state-space matrices of both reduced order models $\widetilde{\mathscr{K}_{\{1.8\}}}$ and $\widetilde{\mathscr{K}}_{\{1.8,0.4\}}$, are given in (54).

As discussed in Section 3.3 (and presented in Table 1), the radiation impulse frequency response $K(j \omega)$ has some particular properties which, ideally, should be retained by the approximate model. To further analyse these characteristics, the pole-zero map of both $\widetilde{K}_{\{1.8\}}$ (lightblue) and $\widetilde{K}_{\{1.8,0.4\}}$ (dark-blue), are shown in Fig. 13. Note, from Fig. 13, that both models have a zero at the origin, consistent with the first property listed in Table 1 . The second property (strictly proper) is automatically guaranteed by the structure of (49). Furthermore, the inputoutput stability is assured by the optimisation process (51), since the set of desired eigenvalues $\Sigma_{k}$ is contained in $\mathbb{C}^{-}$.

Remark 13. Several reduction methods, such as (Perez and Fossen, 2009; Pérez and Fossen, 2008) and (Hatecke, 2015), cannot guarantee input-output stability inherently with the approximation strategies proposed. In fact, if the identified model has a set of unstable poles,
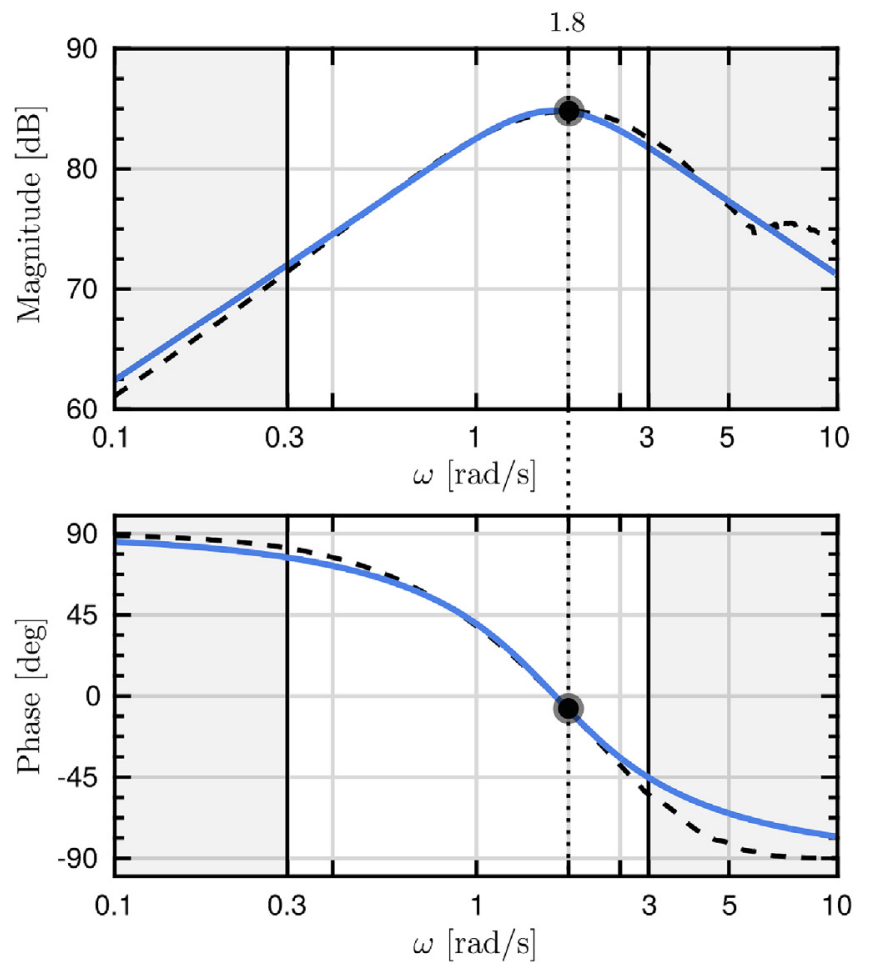

Fig. 11. Bode diagram of the radiation impulse frequency response for the device computed with NEMOH (dashed-black) and the reduced order model frequency response (solid-blue) considering one interpolation point (specified on top of figure). The white area indicates the region of frequencies considered for the approximation. (For interpretation of the references to colour in this figure legend, the reader is referred to the Web version of this article.)

the suggested solution is to "flip" the set, by changing the sign of the real-part of each pole. In contrast, stability can be always guaranteed for the moment-matching based approximation, since one can always choose a stable set of eigenvalues $\Sigma_{k}$ for (49) using $G_{k}$. In fact, this ensures the internal stability of (49), which is a stronger result.

Another key physical property of the radiation system, listed in Table 1, is passivity. Radiation forces are inherently passive: they cannot create energy, but rather dissipate it. After computing several approximate models with a different number of interpolation points and different frequency ranges, numerical simulations suggest that the models obtained are virtually exclusively passive, though the computational process does not explicitly ensure passivity. Note that both parametric models in (54) presented for these numerical examples, are passive.

Nevertheless, if required, a nonlinear constraint can be added to (51) to explicitly secure passivity: the optimisation process can be constrained so that the transfer function $\widetilde{K}(s)(50)$ is positive-real ${ }^{3}$.

Remark 14. Note that the family of reduced order models (49) can be made passive by choosing $G_{k}$ following the theoretical formulation developed in (Astolfi, 2010), to achieve moment-matching with a passivity constraint. However, in this study, $G_{k}$ is considered to assign the eigenvalues of (49) using the optimisation process described in (51).

To illustrate that the models defined in (54) are passive, the Nyquist diagram of $\widetilde{K}_{\{1.4,0.8\}}(j \omega)$ is shown in Fig. 14, where it can be appreciated that the real-part of $\widetilde{K}_{\{1.4,0.8\}}(j \omega)$ is always positive.

Fig. 15 illustrates the time-domain response of the reduced order model $\widetilde{\mathscr{K}_{\{1.8,0.4\}}}$, for a regular wave input, with a frequency of $\omega=0.8$ $[\mathrm{rad} / \mathrm{s}]$. It can be appreciated that the output of the obtained model

\footnotetext{
${ }^{3} \widetilde{K}(s)$ is said to be positive real if the poles of $\widetilde{K}(s)$ have zero or negative real-part and $\mathfrak{R}\{\widetilde{K}(j \omega)\} \geq 0 \forall \omega$ (Khalil, 1996).
} 

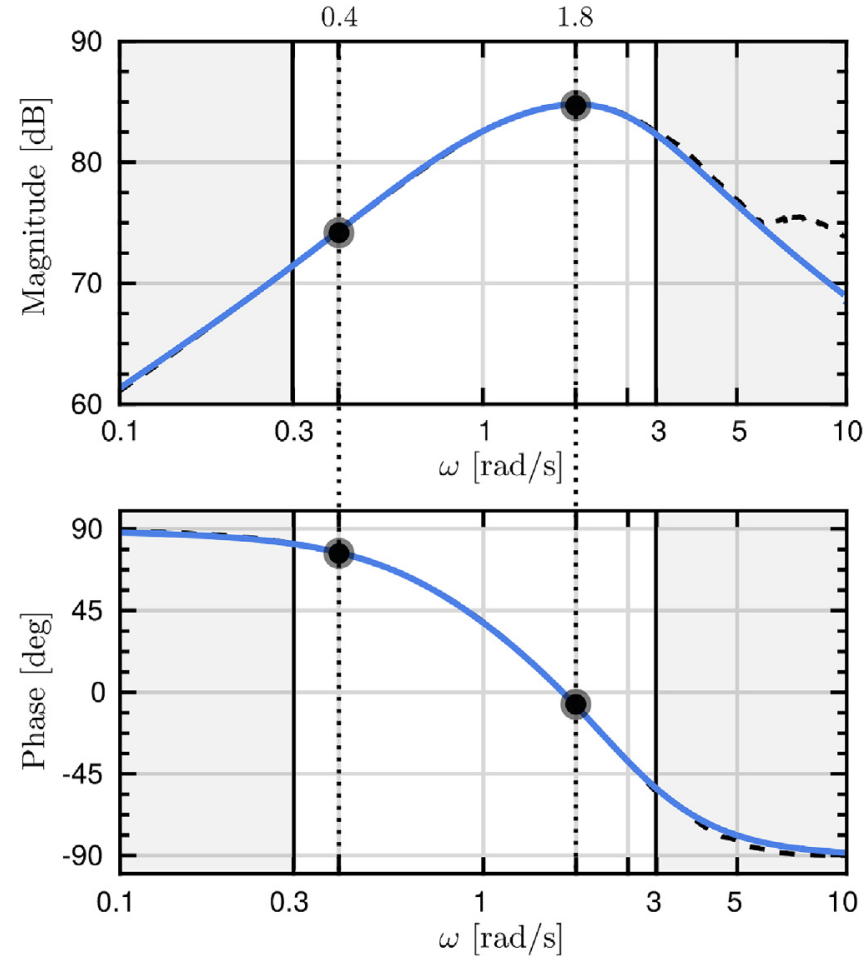

Fig. 12. Bode diagram of the radiation impulse frequency response for the device computed with NEMOH (dashed-black) and the reduced order model (solid-blue) frequency response, considering two interpolation points (specified on top of figure). The white area indicates the region of frequencies considered for the approximation. (For interpretation of the references to colour in this figure legend, the reader is referred to the Web version of this article.)

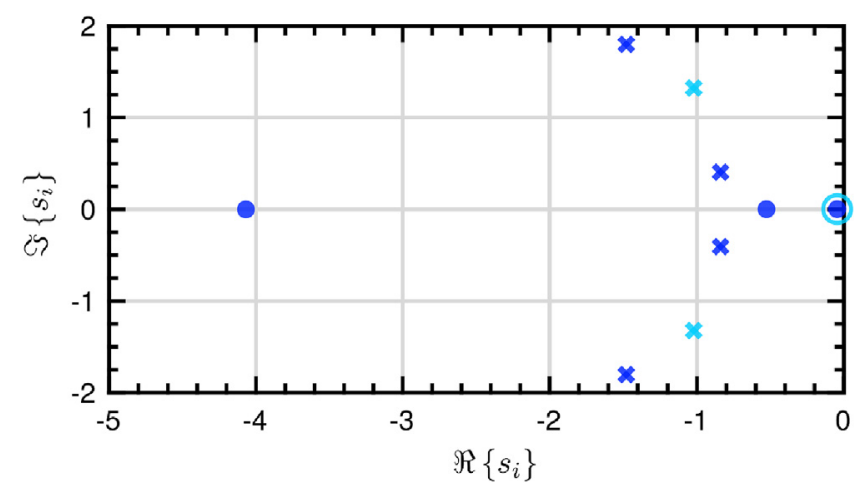

Fig. 13. Pole-zero map of the reduced order models of the impulse response kernel, using one (light-blue) and two (dark-blue) interpolation points. (For interpretation of the references to colour in this figure legend, the reader is referred to the Web version of this article.)

coincides with the desired steady-state response. To expose the difference between selecting one and two interpolation points, Fig. 16 presents the output of both $\widetilde{\mathscr{K}_{\{1.8\}}}$ and $\widetilde{\mathscr{K}_{\{1.8,0.4\}}}$, excited with the same input frequency of $0.8[\mathrm{rad} / \mathrm{s}]$. A slight difference between the target output and the response of $\mathscr{K}_{\{1.8\}}$ (dashed-blue) can be noticed, both in amplitude and phase, whilst the response of the reduced order model interpolating two frequencies $\widetilde{\mathscr{K}_{\{1.8,0.4\}}}$ (solid-blue) perfectly coincides with the desired response. To further illustrate the quality of the timedomain response of the approximated models, Fig. 17 depicts the target radiation impulse response (computed from BEM data), and the impulse response of both reduced order models $\widetilde{\mathscr{K}}_{\{1.8\}}$ and $\mathscr{\mathscr { K }}_{\{1.8,0.4\}}$.

Finally, and to conclude this section on numerical examples of a spherical device, Fig. 18 depicts the approximation error for several

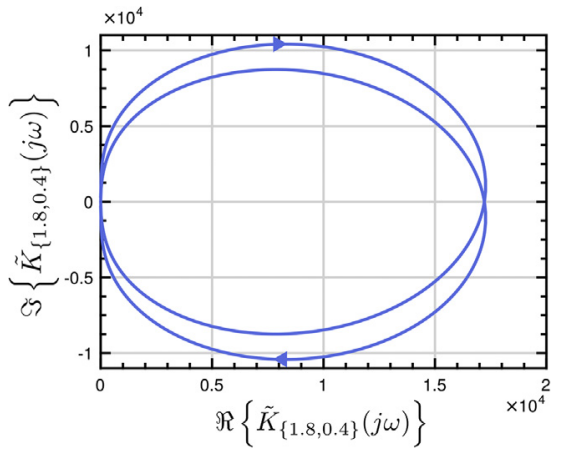

Fig. 14. Nyquist diagram for $\widetilde{K}_{\{1.4,0.8\}}(j \omega)$.

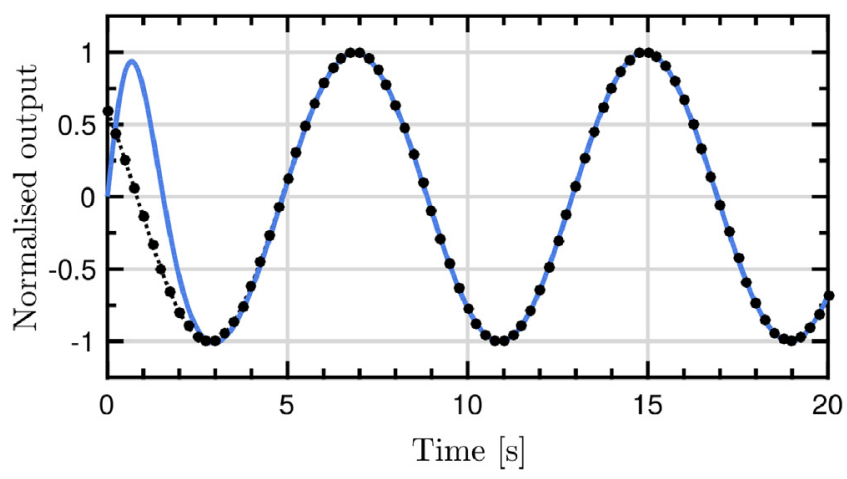

Fig. 15. Comparison between the time-domain output of the reduced order model using two interpolation points (solid-blue) and the frequency response of the radiation kernel of the device (dotted-black), for an input frequency of $\omega=0.8$ [rad]. The amplitude of the output has been normalised to present results between -1 and 1 . (For interpretation of the references to colour in this figure legend, the reader is referred to the Web version of this article.)

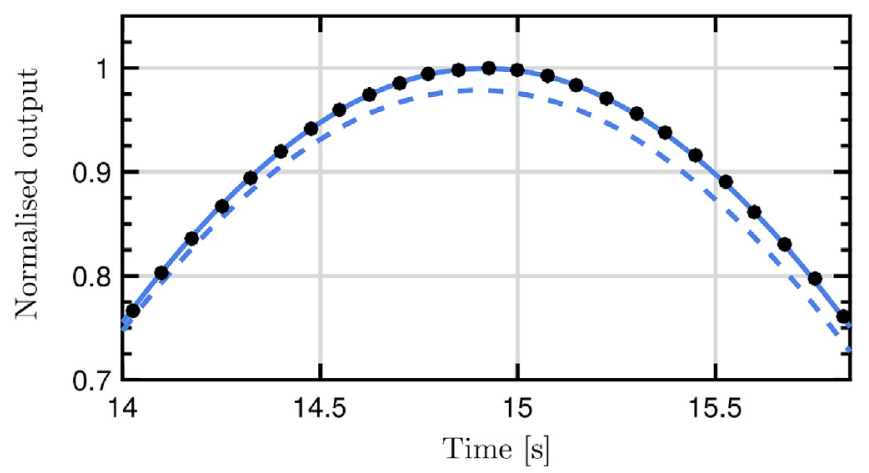

Fig. 16. Comparison between the time-domain output of the reduced order model using one (dashed-blue) and two (solid-blue) interpolation points and the frequency response of the radiation kernel of the device (dotted-black), for an input frequency of $\omega=0.8$ [rad]. The amplitude of the output has been normalised to present results between -1 and 1 . (For interpretation of the references to colour in this figure legend, the reader is referred to the Web version of this article.)

models with different orders $\nu$ (i.e, different number of interpolation points). The error is given in terms of the Mean Absolute Percentage Error (MAPE) (Armstrong and Collopy, 1992), between the target frequency-domain data and the reduced order model frequency response, in the frequency range defined for the approximation. The monotonically decreasing model approximation error is evident in Fig. 18: increasing the order of the radiation impulse response approximated model $(\nu)$ decreases the error monotonically, which is another desired property that a suitable identification algorithm should have, as discussed in Section 1. This gives confidence that each model is optimal for 

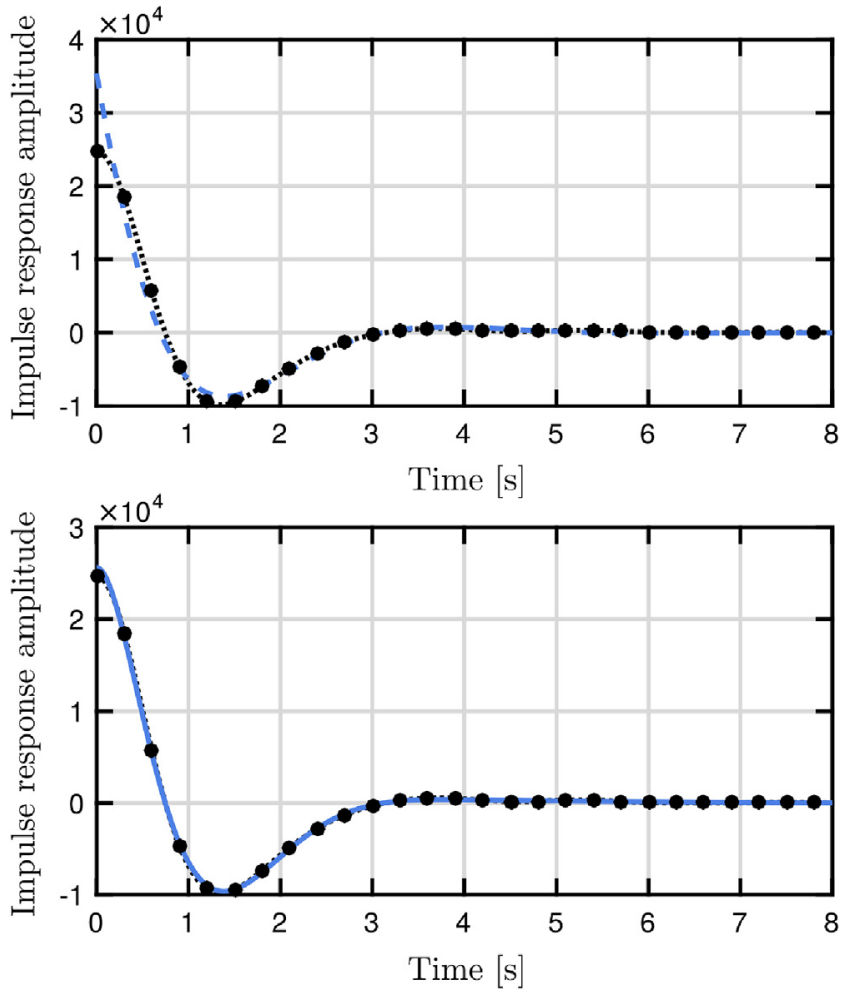

Fig. 17. Comparison between the radiation impulse response computed from BEM data (dotted-black) and the impulse response of $\widetilde{\mathscr{K}}\{1.8\}$ (top, dashed-blue) and $\widetilde{\mathscr{K}}_{\{1.8,0.4\}}$ (bottom, solid-blue). (For interpretation of the references to colour in this figure legend, the reader is referred to the Web version of this article.)

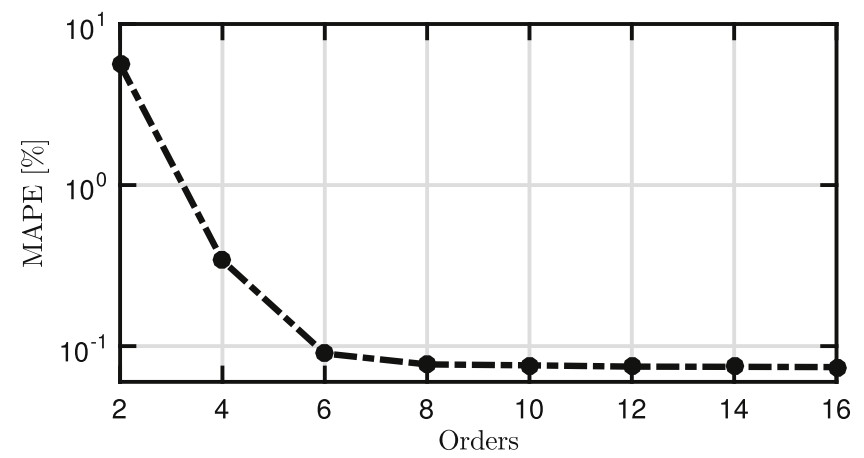

Fig. 18. Approximation error (MAPE) between the target frequency response and the reduced order model frequency response, for different model approximation orders $\nu$.

a given order, while allowing an appropriate order to be chosen judiciously.

\subsection{A more geometrically complex example: the CETO device}

In this subsection, the proposed moment-matching based strategy is applied to a more complex geometry: the CETO device (Penalba et al., 2017a). The choice of such device is merely justified by the complexity of its multimodal frequency response. In the following, the effectiveness of the moment-matching strategy on such a multimodal device is illustrated, by performing an identification of its radiation kernel frequency response.

For this application case, the input frequency range has been set to $\omega_{l}=0.2[\mathrm{rad} / \mathrm{s}]$ and $\omega_{u}=4[\mathrm{rad} / \mathrm{s}]$, to further demonstrate the versatility of the strategy when considering a different frequency space.

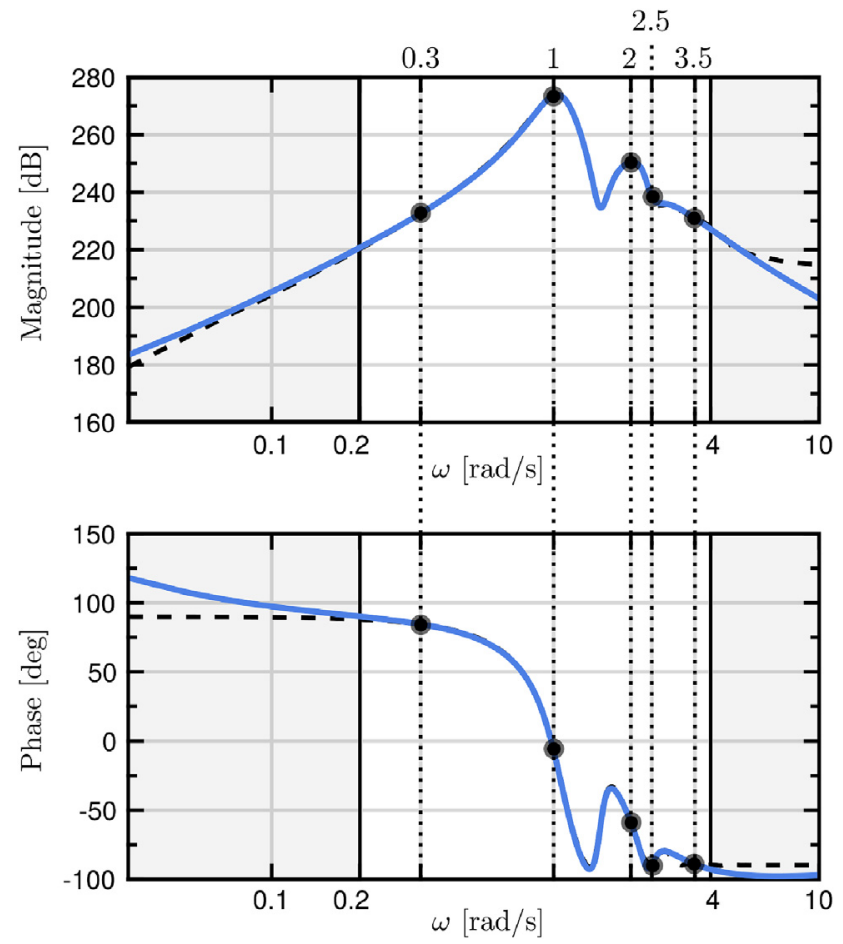

Fig. 19. Bode diagram of the radiation impulse frequency response for the CETO device computed with $\mathrm{NEMOH}$ (dashed-black) and the reduced order model (solid-blue) frequency response, considering five interpolation points (specified on top of figure). The white area indicates the region of frequencies considered for the approximation. (For interpretation of the references to colour in this figure legend, the reader is referred to the Web version of this article.)

Regarding the order of the parametric approximation, five frequencies have been chosen as interpolation points for the moment-matching strategy. Particularly, the selected frequencies are $\{0.3,1,2,2.5,3.5\}$ $[\mathrm{rad} / \mathrm{s}]$. Note that such a choice is not arbitrary: the frequencies $\{1,2,2.5\}$ $[\mathrm{rad} / \mathrm{s}]$ correspond to each peak (mode) of the radiation kernel frequency response illustrated in Fig. 19, while $\{0.3,3.5\}[\mathrm{rad} / \mathrm{s}]$ represent a low and high frequency component, respectively. The frequency response of the obtained moment-based parametric model can be appreciated in Fig. 19. It can be acknowledged that the strategy effectively provides a suitable parametric approximation when considering more geometrically complex devices, successfully fitting a multimodal frequency response, exploiting a sensible choice of the interpolation points.

\section{Conclusions}

In this study, a method to obtain a finite-order hydrodynamic model from frequency-domain data for both the force-to-motion, and the radiation force impulse response transfer function, is proposed, based on recent advances in model order reduction by moment-matching. This formulation allows the user to exactly match the steady-state behaviour of the device under analysis at key frequencies, such as the resonant frequency, retaining important physical properties of the studied WEC, such as input-output stability. Moreover, the moment-matching strategy determines an eigenvalue set to minimise the frequency response error of the approximating model. The different characteristics of the proposed approximation scheme are illustrated with several numerical examples, showing the efficacy of the moment-matchingbased approach. In these numerical examples, the approximation error is shown to be monotonically decreasing with increasing model order, depicting a robust algorithm to obtain parametric hydrodynamic models. Future work will exploit this moment-matching methodology 
to develop reduced order models for arrays of wave energy devices: since the order of the obtained model does not depend on the number of inputs (or outputs), an array of $N$ devices can be described by just a few states, which is potentially beneficial to simulation, estimation, and real-time control of wave energy converter farms. Furthermore, a MATLAB toolbox, to obtain finite-order hydrodynamic models using moment-matching, has been developed, and is available from the Centre for Ocean Energy Research website (Centre for Ocean Energy Research).

\section{Acknowledgment}

The authors are sincerely grateful to Prof. Alessandro Astolfi and Dr. Giordano Scarciotti from Imperial College London, for useful discussions on the moment-based theory. The authors would also like to thank Markel Penalba, for providing the NEMOH data used to numerically illustrate the proposed strategy. This material is based upon works supported by Science Foundation Ireland under Grant no. 13/IA/1886.

\section{References}

Antoulas, A.C., 2005. Approximation of Large-scale Dynamical Systems. SIAM. Armstrong, J.S., Collopy, F., 1992. Error measures for generalizing about forecasting methods: empirical comparisons. Int. J. Forecast. 8 (1), 69-80.

Astolfi, A., 2010. Model reduction by moment matching for linear and nonlinear systems. IEEE Trans. Automat. Contr. 55 (10), 2321-2336.

Babarit, A., Delhommeau, G., 2015. Theoretical and numerical aspects of the open source BEM solver NEMOH. In: 11th European Wave and Tidal Energy Conference, Nantes.

Bacelli, G., Ringwood, J.V., 2015. Numerical optimal control of wave energy converters. IEEE Transactions on Sustainable Energy 6 (2), 294-302.

Bertram, V., Pereira, R., Landrini, M., 2001. An enhanced nonlinear strip method for seakeeping analysis. In: Proceedings 16th International Workshop on Water Waves and Floating Bodies, Japan.

Brewer, J., 1978. Kronecker products and matrix calculus in system theory. IEEE Trans. Circ. Syst. 25 (9), 772-781.

Centre for Ocean Energy Research, http://www.eeng.nuim.ie/coer/.

Cummins, W., 1962. The Impulse Response Function and Ship Motions. Tech. rep., DTIC Document. .

N. Faedo, S. Olaya, J. V. Ringwood, Optimal control, MPC and MPC-like algorithms for wave energy systems: an overview, IFAC J. Syst. Contr.

N. Faedo, G. Scarciotti, A. Astolfi, J. V. Ringwood, Energy-maximising control of wave energy converters using a moment-domain representation, Contr. Eng. Pract., (Under Review).

Falnes, J., 2002. Ocean Waves and Oscillating Systems: Linear Interactions Including Wave-energy Extraction. Cambridge university press.

Hasselmann, K., 1973. Measurements of wind wave growth and swell decay during the joint north sea wave project (JONSWAP). Dtsch. Hydrogr. Z. 8, 95.

Hatecke, H., 2015. The impulse response fitting and ship motions. Ship Technol. Res. 62 (2), 97-106.

Holappa, K., Falzarano, J., 1998. Application of extended state space to nonlinear ship rolling. Ocean Eng. 26 (3), 227-240.

Jordán, M.A., Beltrán-Aguedo, R., 2004. Optimal identification of potential-radiation hydrodynamics for moored floating structuresa new general approach in state space.
Ocean. Eng. 31 (14), 1859-1914.

Khalil, H.K., 1996. Noninear Systems, Prentice-Hall. New Jersey.

Kristiansen, E., Hjulstad, Å., Egeland, O., 2005. State-space representation of radiation forces in time-domain vessel models. Ocean Eng. 32 (17), 2195-2216.

McCabe, A., Bradshaw, A., Widden, M., 2005. A Time-domain Model of a Floating Body Using Transforms. 6th European Wave and Tidal Energy Conference, Glasgow, pp. 281-288.

Newman, J., Lee, C.-H., 2002. Boundary-element methods in offshore structure analysis. J. Offshore Mech. Arctic Eng. 124 (2), 81-89.

$\emptyset$, Y., Rogne, T., Moan, Ersdal, S., 2014. Identification of passive state-space models of strongly frequency dependent wave radiation forces. Ocean Eng. 92, 114-128.

Ochi, M.K., Hubble, E.N., 1976. Six-parameter Wave Spectra, in: Coastal Engineering, vol. 1977. pp. 301-328.

Ogilvie, T.F., 1964. Recent progress toward the understanding and prediction of ship motions. In: 5th Symposium on Naval Hydrodynamics, vol. 1. pp. 2-5 Bergen, Norway.

Penalba, M., Kelly, T., Ringwood, J.V., 2017a. Using Nemoh for Modelling Wave Energy Converters: a Comparative Study with Wamit.

Penalba, M., Touzón, I., Lopez-Mendia, J., Nava, V., 2017b. A numerical study on the hydrodynamic impact of device slenderness and array size in wave energy farms in realistic wave climates. Ocean Eng. 142, 224-232.

Pérez, T., Fossen, T.I., 2008. Time-vs. frequency-domain identification of parametric radiation force models for marine structures at zero speed. Model. Identif. Control 29 (1), 1-19.

Perez, T., Fossen, T.I., 2009. A matlab toolbox for parametric identification of radiationforce models of ships and offshore structures. Model. Identif. Control 30 (1), 1.

Perez, T., Lande, O., 2006. Time-domain models of marine surface vessels for simulation and control design based on seakeeping computations. In: IFAC Conference on Manoeuvring and Control of Marine Craft MCMC. IFAC, Lisbon.

J. V. Ringwood, M. Alexis, N. Faedo, F. Fusco, On the sensitivity and robustness of wave energy control systems, IEEE Trans. Contr. Syst. Technol.

Roessling, A., Ringwood, J.V., 2015. Finite order approximations to radiation forces for wave energy applications. Renewable Energies Offshore 359.

Scarciotti, G., Astolfi, A., 2015. Characterization of the moments of a linear system driven by explicit signal generators. In: American Control Conference (ACC), 2015. IEEE, pp. 589-594.

Scarciotti, G., Astolfi, A., 2016a. Model reduction by matching the steady-state response of explicit signal generators. IEEE Trans. Automat. Contr. 61 (7), 1995-2000.

Scarciotti, G., Astolfi, A., 2016b. Moment-based discontinuous phasor transform and its application to the steady-state analysis of inverters and wireless power transfer systems. IEEE Trans. Power Electron. 31 (12), 8448-8460.

Scarciotti, G., Astolfi, A., 2017a. Nonlinear model reduction by moment matching. Foundations and Trends in Systems and Control 4 (3-4), 224-409.

Scarciotti, G., Astolfi, A., 2017b. Data-driven model reduction by moment matching for linear and nonlinear systems. Automatica 79, 340-351.

Sutulo, S., Soares, C.G., 2005. An implementation of the method of auxiliary state variables for solving seakeeping problems. Int. Shipbuild. Prog. 52 (4), 357-384.

Taghipour, R., Perez, T., Moan, T., 2008. Hybrid frequency-time domain models for dynamic response analysis of marine structures. Ocean Eng. 35 (7), 685-705.

Unneland, K., 2007. Identification and Order Reduction of Radiation Force Models of marine Structures. Ph.D. thesis. NTNU.

Van Loan, C.F., 2000. The ubiquitous kronecker product. J. Comput. Appl. Math. 123 (1), 85-100.

Wazwaz, A.-M., 2011. Volterra integro-differential equations. In: Linear and Nonlinear Integral Equations. Springer, pp. 175-212.

Xia, J., Wang, Z., Jensen, J.J., 1998. Non-linear wave loads and ship responses by a timedomain strip theory. Mar. Struct. 11 (3), 101-123.

Yu, Z., Falnes, J., 1995. State-space modelling of a vertical cylinder in heave. Appl. Ocean Res. 17 (5), 265-275. 